Research paper

\title{
A Fully Developed Flow Thermofluid Model for Topology Optimization of 3D-Printed Air-Cooled Heat Exchangers
}

\author{
Jan H. K. Haertel ${ }^{\mathrm{a}}$, Gregory F. Nellis ${ }^{\mathrm{b}}$ \\ ${ }^{a}$ Department of Energy Conversion and Storage, Technical University of Denmark, Frederiksborgvej \\ 399, 4000 Roskilde, Denmark, jhkh@dtu.dk \\ ${ }^{\mathrm{b}}$ Department of Mechanical Engineering, University of Wisconsin-Madison, 1500 Engineering Drive, \\ Madison, WI 53706, USA, gfnellis@engr.wisc.edu \\ * Corresponding author
}

\begin{abstract}
In this work, density-based topology optimization is applied to the design of the air-side surface of dry-cooled power plant condensers. A topology optimization model assuming a steady-state, thermally and fluid dynamically fully developed internal flow is developed and used for this application. The conductance of the heat exchanger is maximized for a prescribed pressure drop and prescribed air-side temperature change across the heat exchanger. Polymer with infilled thermally conducting metal filaments is considered as the heat exchanger material which allows cost effective additive manufacturing techniques to be used to fabricate the obtained designs. Parametric studies are presented that analyze the effect of the material thermal conductivity and the heat exchanger unit cell height on the system's performance. The designs obtained from topology optimization are benchmarked against a simple optimized slot channel model in order to demonstrate the superior performance of the topology optimized designs. Thus, this work demonstrates the usefulness of topology optimization to fully exploit the design freedom afforded by additive manufacturing technologies.
\end{abstract}

\section{Introduction}

High performance air-side heat transfer surfaces are required by many applications. The work presented here was motivated specifically by the need to transition heat rejection systems for power plants from water-cooled to air-cooled (referred to subsequently as dry-cooled). Dry-cooling of power plants eliminates their water consumption, thus mitigating their environmental impact and providing a higher flexibility for siting options since no large local water source is needed. Currently, water-cooled systems are preferred due to their low cost and compact design; however, power plants are currently the single largest users of fresh water in the US [1] and this is increasingly becoming an issue in several regions of the country. The penalty associated with dry-cooling 
is lower efficiency due to higher heat rejection temperatures (the dry-bulb vs the wet-bulb temperature) and the lower air-side heat transfer coefficient as well as comparatively high required fan power. These disadvantages have thus far prevented the wide spread use of dry-cooled condensers. Improving the air-side heat transfer surface while keeping the fan power low is crucial for dry-cooled heat exchangers to become economically competitive. In this work, density-based topology optimization is applied to address this optimization challenge.

The application of topology optimization to the design of thermofluid systems is an active area of research. Topology optimization allows a systematic optimization of these nonlinear systems as opposed to classical methods such as trial and error or intuition-based engineering. Therefore, topology optimization can lead to reduced development time and unanticipated and non-intuitive designs can be identified. Nevertheless, the performance increase of the topology optimized designs can result in more complex heat exchanger surfaces than when applying classical design methods. These complex surfaces might not be easily manufactured using conventional manufacturing techniques; however, they can be fabricated using additive manufacturing methods, especially as those methods become increasingly mature. Thus, topology optimization techniques are critical in order to fully exploit the design freedom that these technologies provide. An additive manufacturing or 3D printing method that is being considered for the fabrication of dry-cooled condensers is Fused Layer Modeling (FLM) where polymer material is extruded through a nozzle to build up a structure layer by layer. Using this method, complex surfaces can be fabricated from a relatively cheap polymer in large quantities and with cheap equipment. High throughput can be obtained using several nozzles in parallel. As a result, the FLM process is fundamentally cost-effective for larger structures such as dry-cooled condensers. The general applicability of polymers as heat exchanger and heat sink material has been demonstrated successfully in different applications [2]. A significant drawback of using polymers as heat exchanger material is the thermal conductivity which is approximately 100 times lower than metal. However, this limitation can be overcome by using fillers such as thermally conductive carbon fibers or metal filaments to increase the effective conductivity of the resulting composite.

Density-based topology optimization [3] determines whether to put solid or void at each point within a design domain in order to maximize a given objective under specified constraints. For this purpose, a density field is introduced that takes the value of 0 for solid and 1 for void which, for this application, corresponds to heat exchanger material (0) or fluid passage (1). Relaxing this optimization problem to density values between 0 and 1 allows for the use of gradient-based optimization methods, which are necessary to efficiently tackle topology optimization problems that typically involve large numbers of design variables. Topology optimization was originally developed for structural mechanics applications and has matured within this field; however it has subsequently been applied to other disciplines such as fluid mechanics [4] and photonics [5].

One application of topology optimization in heat transfer is pure heat conduction problems, as described in $[3,6,7]$. In addition to pure two dimensional heat conduction, out of plane convection with a constant heat transfer coefficient is considered in various publications: For example Sigmund [8] applied density-based topology optimization to the design of multiphysics actuators. Later works also included in-plane convective heat transfer to an ambient fluid either assuming a constant heat transfer coefficient [9-11] or using a surrogate model for the heat transfer coefficient [12]. This modeling approach was extended to 3D conduction problems with convection to an ambient fluid assuming a constant heat transfer coefficient using density-based [13] and 
level set topology optimization [14]. Soprani et al. [15, 16] applied density-based topology optimization to the thermal integration of a thermoelectric cooler by designing the heat rejection path to convectively cooled boundaries with prescribed heat transfer coefficient. To overcome these simplifications regarding the details of the convective heat transfer process to the ambient fluid, more recent works included heat conduction in the solid domain and explicit modeling of heat transfer in the fluid within the topology optimization. Different works presented forced convection thermofluid topology optimization models using density-based topology optimization [17-28] and level set boundary expressions [29]. Dede [17] presents 2D and 3D heat conduction problems as well as a 2D thermofluid model with uniform heat production rate in the modeled domain and a fixed Reynolds number of 50 at the fluid inlet. The optimization of different 2D modeled heat dissipating structures is presented by [18] and [19, 20] apply 2D topology optimization to the design of water-cooled microchannel heat sinks where [20] limits the fluid problem to Stokes flow. Marck et al. [21] present pure fluid 2D optimization problems as well as a $2 \mathrm{D}$ thermofluid topology optimization model for a pipe with constant wall temperature and report a Reynolds number of 3 in the thermofluid problem. A 2D thermofluid heat exchanger topology optimization model under constant input power is described in [22] where the case of a temperature dependent and temperature independent heat source within the design domain is considered and a maximum Reynolds number of 100 is prescribed. Similar models are used in [23] that model the 2D thermofluid problem using the lattice Boltzmann method and report Reynolds numbers of around 10 as well as in [29] that use level set boundary expressions for the topology optimization and generate 2D and 3D optimized designs with a maximum Reynolds number of 800 for the 2D case. The 2D modeling approach of the above mentioned thermofluid works was extended by [24] and later [25] to a pseudo 3D model where a 2D conductive base layer in which the heat generation occurs interacts with the 2D thermofluid optimization layer. Qian et al. [26] presented topology optimization for a 2D thermofluid system under a tangential thermal gradient constraint generating designs for Reynolds numbers of 50 and 250. The above mentioned works on thermofluid topology optimization treat laminar flow problems. Kontoleontos et al. [27] use a Spalart-Allmaras model to extend the modeling to turbulent flow; however, the temperature distribution in the solid is ignored in their work. Topology optimization for natural convection problems has only recently been addressed and work has been reported applying density-based topology optimization to 2D models [30] as well as 3D- [31] and large-scale 3D systems [32]. Coffin and Maute used level set-based topology optimization to optimize 3D and 2D transient natural convection systems [33]. The design using topology optimization, manufacturing and subsequent experimental testing of optimized heat sinks was presented for a forced convection 2D Stokes flow thermofluid model [28] and a 2D air-cooled heat sink model considering conduction and side surface convection [34]. Castro et al. [35] use the topology optimization method to design enclosures in which radiation is the dominant heat transfer mechanism. For a more detailed description of the literature on thermal and thermofluid topology optimization, the reader is referred to a very recently published review paper on this topic [36]. An extensive body of literature deals with various other optimization approaches applied to heat exchanger design and a detailed presentation of these works lies outside the scope of this study. Therefore, the readers are referred to an exemplary work presenting stochastic optimization of air-cooled heat exchangers where the heat transfer is modeled based on correlations [37] and a review paper on CFD applications in heat exchanger design [38] that also covers literature on optimization in this context.

The 2D models that explicitly consider heat transfer in the fluid [17-30] have in common that the fluid flow is within the plane of the design domain. The model presented in this study presents for the first time an approach 
where a flow is modeled that is perpendicular to the design domain; this is considered one of the main contributions of this work. One specific benefit of the presented model could be the combination with existing works on thermofluid topology optimization modeling the fluid flow within the design domain plane; hence creating a cross-flow heat exchanger optimization model with explicit modeling of both fluids flowing perpendicular to each other. Another advantage of assuming a fluid dynamically fully developed flow is the simplification of the Navier-Stokes equation to a linear partial differential equation (PDE). This circumvents problems with the increasing nonlinearity of the Navier-Stokes equations that accompany increasing Reynolds number and have so far limited thermofluid density-based topology optimization to Stokes flow or laminar flow with relatively low Reynolds numbers except for [27] that ignore the temperature distribution in the solid. Thus, the approach presented here extends the range of applicability of thermofluid topology optimization since it can be applied to problems with arbitrary Reynolds numbers within in the laminar flow regime provided that one can assume fully developed flow. Extension of the approach to even larger Reynolds number may be possible with a suitable turbulence closure model. Moreover, the above mentioned works, except for $[13,15,16]$ which do not explicitly model the fluid flow, treat rather academic heat sink or heat exchanger problems whereas the optimizations in this study are conducted for specific "real world" operating conditions associated with drycooled power plant condensers, even though the optimization is accomplished using some simplifying assumptions. However, it should be noted that the presented modeling approach is by no means limited to the specific application considered in this work but can be applied to arbitrary thermofluid systems in which the fully developed flow assumption is reasonable.

Parametric studies are conducted using the optimization model in order to analyze the influence of the effective conductivity of the filled polymer composite as well as the unit cell height used in the heat exchanger structure on different measures of the system performance. This analysis of the topology optimization results on a system level in the context of a specific heat exchanger design problem is considered another main contribution of this work as current literature on thermofluid topology optimization focuses mainly on the topology optimization implementation and exemplary generation of a few optimized designs. The topology optimized designs generated in this work are compared to a simple, optimized slot channel model in order to demonstrate the improvement in performance afforded by the topology optimization. This study provides some confirmation of the usefulness of topology optimization to exploit the design freedom that is provided by additive manufacturing techniques.

\section{Heat exchanger model}

\subsection{General design and modeling of the heat exchanger}

The heat exchanger is configured in a cross-flow arrangement which is typical for gas-to-liquid heat exchangers. This is done to create a relatively large frontal area and short air-channels, which allows a large surface area for heat transfer while keeping the air-side pressure drop low. This arrangement is also convenient for headering the two fluids. The heat exchanger macrostructure consists of a large array of unit cells; the macrostructure and a single unit cell are schematically depicted in Fig. 1. Within the unit cell, there is substantial design freedom relative to the geometry of the air-side heat transfer surface and the use of heat transfer enhancing structures. However, walls with a certain minimal thickness separating the water and air flows are needed to ensure a 
waterproof design and vertical walls between the unit cells are required periodically in order to ensure mechanical stability of the macrostructure.

Since topology optimization is computationally expensive due to the requirement of typically hundreds of design iterations before convergence to an optimized design, the unit cell is modeled in 2D to reduce the computational complexity. This modeling approach considers the unit cell cross section perpendicular to the air flow, as shown in Fig. 2. Colored in grey are the required walls separating the water and air flow at the top and bottom of the unit cell as well as the required side walls of the unit cell. Within the blue inner area, the distribution of material can be freely chosen in order to shape the air channels using heat transfer enhancing structures. To further reduce the computational resources required, only a part of the unit cell's cross section is modeled, as shown in Fig. 2. By exploiting symmetry on the left, right, and bottom of the modeled domain, a periodic design representative of the entire unit cell can be obtained by optimizing a relatively small fraction of the cell. Only minor adjustments to this periodic design would need to be made in the regions directly adjacent to the side walls of the unit cell to avoid channeling. It should be noted that the water-side heat-transfer is not modeled explicitly in this approach and instead a water temperature is prescribed as the boundary condition on the outer side of the wall. This further reduces the model's computational demand and is justified by the fact that the water-side heat transfer coefficient is typically quite large and the dominant thermal resistance in a dry-cooled condenser will be on the air-side.
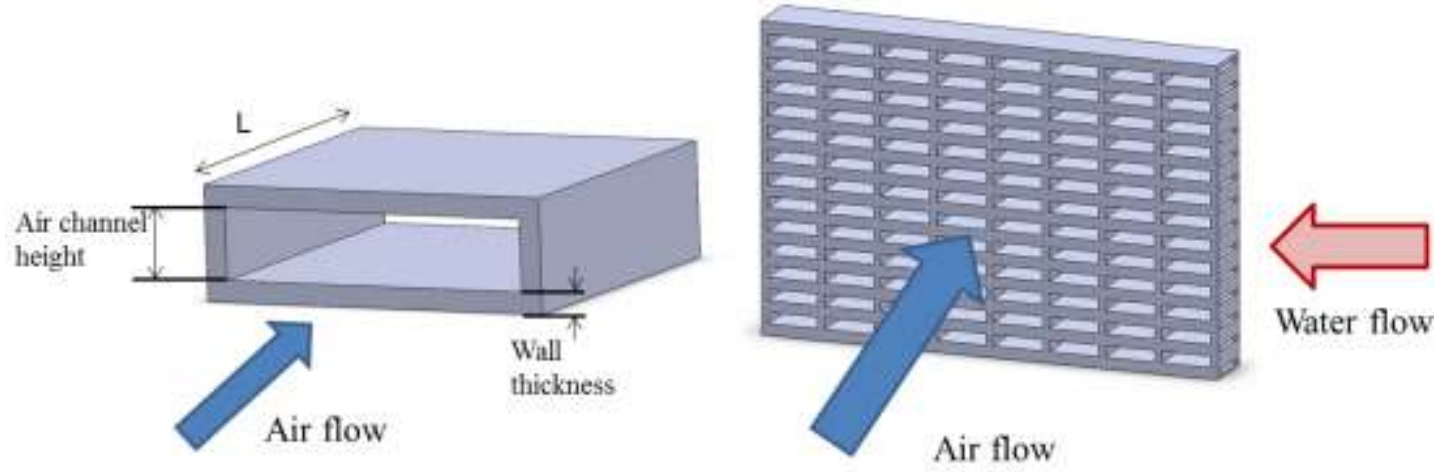

Fig. 1. Air-side heat exchanger unit cell (left) shown without heat transfer enhancing structures and macrostructure (right) consisting of a large array of unit cells. 


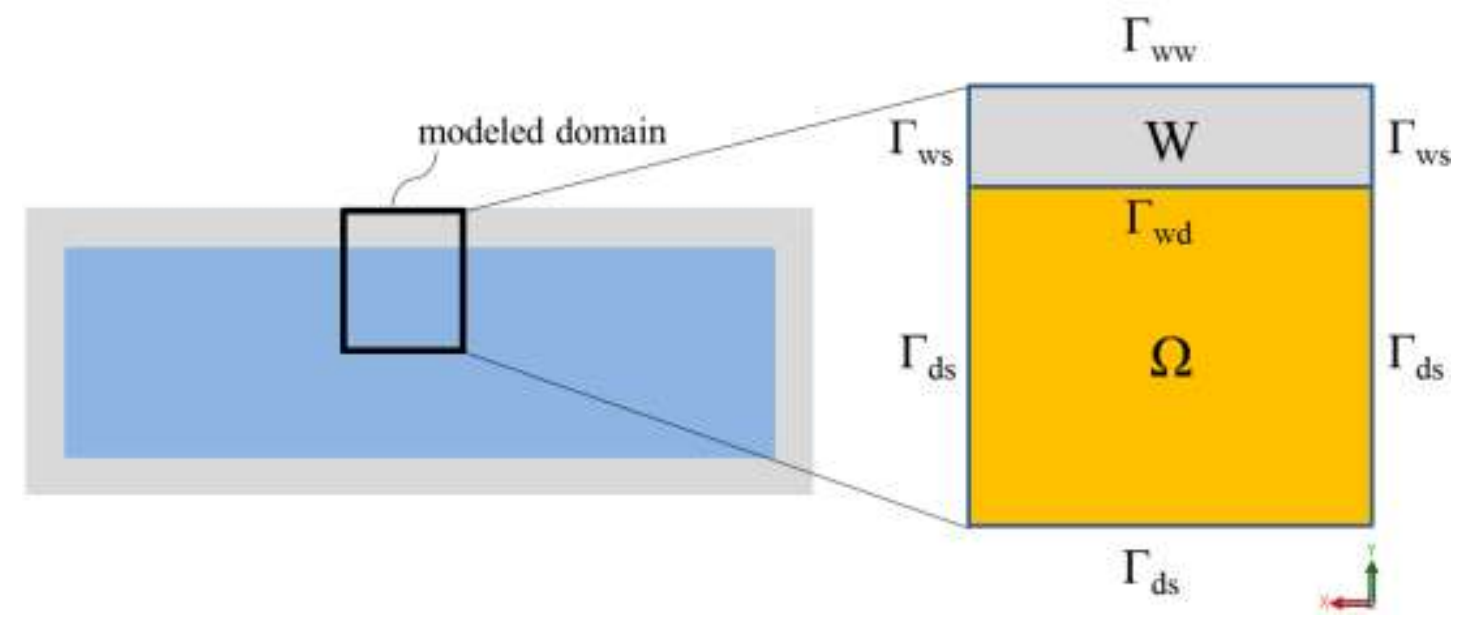

Fig. 2. Illustration of frontal view on heat exchanger unit cell (left) and zoomed in view on the domain modeled for the optimization (right). The domains $\Omega$ (design domain) and $W$ (wall) are indicated and the definition of the different domain boundaries $\Gamma_{i j}$ is given.

\subsection{Governing equations}

Throughout this study, an incompressible, steady-state, and laminar flow is assumed within the heat exchanger. It is important to note that the laminar flow assumption depends on the geometry of the generated heat exchanger designs. Therefore, this assumption needs to be verified once the optimized designs are generated which is discussed in chapter 4 and Appendix A.

\section{Fluid dynamics modeling}

The Navier-Stokes equation for an incompressible, pressure-driven, fully developed internal flow in the $z$ direction is given by:

$\mu\left(\frac{\partial^{2} w}{\partial x^{2}}+\frac{\partial^{2} w}{\partial y^{2}}\right)=\frac{d p}{d z}$

where $w$ is the fluid velocity in $z$-direction, $\mu$ the dynamic viscosity of the fluid, and $\frac{d p}{d z}$ the pressure gradient in $z$ direction. In fluid flow topology optimization a Brinkman friction term, which is the force exerted on a fluid flowing through an ideal porous medium, is used to penalize fluid flow through the solid area, as described in [4]. The Brinkman friction term is defined as:

$F(\gamma, w)=\bar{\alpha}(1-\gamma) w$

where $\gamma$ is the design variable and $\bar{\alpha}$ is the maximum inverse permeability. The value of $\bar{\alpha}$ should be chosen to be sufficiently large to ensure that the flow through solid area is negligibly small; however, if $\bar{\alpha}$ is set to a value that is too high then numerical instability can occur. Usually, a convex interpolation is used to interpret the Brinkman friction term as presented in [4]. However, since simplified linear Navier-Stokes equations are analyzed in this work, a linear interpolation was found to yield good results. Introducing Eq. (2) into Eq. (1), replacing the axial pressure gradient by the pressure drop over the heat exchanger and considering air as the fluid provides: 
$\mu_{\text {air }}\left(\frac{\partial^{2} w}{\partial x^{2}}+\frac{\partial^{2} w}{\partial y^{2}}\right)=\frac{\Delta p_{H E X}}{L}-\bar{\alpha}(1-\gamma) w \quad$ in $\Omega$

where $\Delta p_{H E X}$ is the air-side pressure drop over the heat exchanger unit cell, which is set to a fixed value during the optimization, and $L$ is the length of the unit cell in the flow direction. A no-slip condition is assumed at the wall and symmetry is imposed on the remaining boundaries ${ }^{1}$ as stated above. This leads to the following set of boundary conditions:

$$
\begin{array}{ll}
w=0 & \text { on } \Gamma_{w d} \\
-\mathbf{n} \cdot(-\nabla w)=0 & \text { on } \Gamma_{d s}
\end{array}
$$

where $\mathbf{n}$ is the normal vector to the boundary, $\nabla$ is the nabla operator, and $\Gamma_{i j}$ are the respective domain boundaries, as specified in Fig. 2.

\section{Heat transfer modeling}

The two dimensional conductive heat transport in the solid filled polymer can be described as follows:

$k_{s}\left(\frac{\partial^{2} T_{s}}{\partial x^{2}}+\frac{\partial^{2} T_{s}}{\partial y^{2}}\right)=0$

where $T_{s}$ is the temperature of the solid and $k_{s}$ is the thermal conductivity of the material. The thermal convection-diffusion equation for thermally and fluid dynamically fully developed internal laminar flow in the $z$ direction [39] is given by:

$w \rho_{f} c_{f} \frac{\partial T_{f}}{\partial z}=k_{f} \frac{\partial^{2} T_{f}}{\partial z^{2}}+k_{f}\left(\frac{\partial^{2} T_{f}}{\partial x^{2}}+\frac{\partial^{2} T_{f}}{\partial y^{2}}\right)+\dot{g}_{v}^{\prime \prime \prime}$

where $T_{f}$ is the fluid temperature, $\rho_{f}$ is the fluid density, $c_{f}$ is the specific heat capacity of the fluid, $k_{f}$ is the conductivity of the fluid, and $\dot{g}_{v}^{\prime \prime \prime}$ is the volumetric rate of viscous dissipation. Neglecting axial conduction and viscous dissipation and expressing the axial temperature gradient as the ratio of the air-side temperature increase to the heat exchanger length leads to the following equation:

$w \rho_{f} c_{f} \frac{\Delta T_{H E X}}{L}=k_{f}\left(\frac{\partial^{2} T_{f}}{\partial x^{2}}+\frac{\partial^{2} T_{f}}{\partial y^{2}}\right)$

where $\Delta T_{H E X}$ is the air-side temperature increase across the heat exchanger unit cell, which is set to a fixed value during the optimization. To allow for a unified representation of the heat transport in the polymer, Eq. (5), and air, Eq. (7), an interpolation for the thermal conductivity is introduced:

\footnotetext{
${ }^{1}$ Both symmetry boundary conditions (BCs) and periodic BCs could be used for this design problem. However, the constraints on the design are less restrictive when using a symmetry BC. E.g., for a very narrow modeled domain only half of a fin (fluid at left side of modeled domain and solid on right side) could be formed using a symmetry BC which would not be possible using a periodic BC. Due to this added flexibility, symmetry BCs where chosen in this work.
} 
$\gamma w \rho_{\text {air }} c_{\text {air }} \frac{\Delta T_{H E X}}{L}=k(\gamma)\left(\frac{\partial^{2} T}{\partial x^{2}}+\frac{\partial^{2} T}{\partial y^{2}}\right) \quad$ where $\boldsymbol{\gamma}=\mathbf{0}$ in $W$ and $\gamma \in[\mathbf{0}, \mathbf{1}]$ in $\Omega$

The axial energy transport term on the left hand side of equation (8) can be interpreted as a velocity dependent heat sink. Through this term, the thermal modeling is coupled to the fluid velocity field which implicitly depends on the density field of the design variable. The convective energy transport term is additionally penalized with a linear interpolation as presented in $[22,26]$. This ensures that the small amount of fluid leakage through the solid domain, which is to some degree unavoidable in density-based topology optimization, does not interact with the thermal modeling. To interpolate between the thermal conductivity of solid and fluid during the optimization process, a RAMP-style function [40] as presented in [30] is used which leads to:

$k(\gamma)=k_{\text {air }} \frac{\gamma\left(\frac{k_{\text {polymer }}}{k_{\text {air }}}\left(1+b_{k}\right)-1\right)+1}{\frac{k_{\text {polymer }}}{k_{\text {air }}}\left(1+b_{k} \gamma\right)}$

where $k_{\text {air }}$ and $k_{\text {polymer }}$ are the respective thermal conductivities of air and polymer and $b_{k}$ is a parameter that controls the convexity of the interpolation. Since the water-side heat transfer is not explicitly modeled, the temperature at the outer boundary of the wall is set to a fixed value. The remaining boundaries are modeled with a symmetry condition as described in section (2.1). This leads to the following set of boundary conditions for equation (8):

$$
\begin{array}{ll}
T=T_{\text {water }} & \text { on } \Gamma_{w w} \\
-\mathbf{n} \cdot(-\nabla T)=0 & \text { on } \Gamma_{d s} \text { and } \Gamma_{w s}
\end{array}
$$

where $T_{\text {water }}$ is the prescribed water temperature.

Thus, the thermofluid problem solved in this work is stated by the equations (3) and (8) as well as the respective boundary conditions given by the equations (4) and (10). As mentioned above, the thermal problem is coupled to the fluid velocity field through the convective energy transport term in $z$-direction. However, it should be noted that there is no back coupling from the thermal to the fluid model since constant material properties are used throughout the optimization.

\section{Topology optimization implementation}

\subsection{Optimization problem}

The optimization objective is to maximize the heat exchanger's conductance $U A$ which is the inverse of the total thermal resistance between water and air. $U A$ is defined in the context of the $2 \mathrm{D}$ model according to:

$U A=\frac{\dot{q}}{\left(T_{\text {water }}-T_{\text {air }, \text { bulk }}\right)}=\frac{\dot{q}}{\Delta T}$

where $\dot{q}$ is the heat transfer rate from the water to the air within the modeled domain, $T_{\text {water }}$ is the prescribed boundary condition, and $T_{\text {air, bulk }}$ is the velocity weighted average air temperature (i.e., the bulk temperature). As described in chapter 2 , the water-side heat transfer is not explicitly modeled in this work, which is why $T_{\text {water }}$ is 
used as an approximation for $T_{\text {water, bulk }}$ in Eq. (11). Thus, the water-side heat transfer resistance is not considered in the optimization which is justified by the fact that the air-side heat transfer resistance is dominant in air-towater heat exchangers. Moreover, the water-side geometry is not changing during the optimization as the design domain is only on the air side of the heat exchanger. $T_{a i r, \text { bulk }}$ can be computed by:

$T_{\text {air }, \text { bulk }}=\frac{1}{A_{\Omega, f l} w_{\text {average }, f l}} \int_{\Omega} \gamma w T d \Omega$

where $A_{\Omega, f l}$ is the flow area of the design domain and $w_{\text {average, } f l}$ is the flow area averaged air velocity within the design domain. However, maximizing the conductance can lead to local optima associated with situations where the temperature difference in the denominator of Eq. (11) approaches 0. Therefore, a slightly modified optimization formulation is used in which the heat transfer rate is maximized and the difference between the air bulk temperature and the water temperature is set as a constraint. This approach corresponds to maximizing the conductance for a fixed value of air-to-water temperature difference, $\Delta T$, and leads to the following optimization problem:

$\max \dot{q}(\boldsymbol{\gamma}, \boldsymbol{s})$

s.t. $\left(T_{\text {water }}-T_{\text {air }, \text { bulk }}(\boldsymbol{\gamma}, \boldsymbol{s})\right)<\left(T_{\text {water }}-T_{\text {air }, \text { bulk }}^{*}\right)$

$r(\gamma, s)=0$

$0 \leq \gamma_{i} \leq 1 \quad$ for $i=1, \ldots, n_{d}$

where $\boldsymbol{\gamma}$ is the vector of the design variables, $\boldsymbol{s}$ is the vector of the state variables of the multiphysics problem described in section 2, $T_{\text {air }, \text { bulk }}^{*}$ is the prescribed bulk air temperature, $r(\gamma, s)$ is the residual of the finite element formulation of the multiphysics problem, and $n_{d}$ is the number of design variables.

\subsection{Density filtering and projection}

Density filtering is used to solve issues with ill-posedness of thermofluid topology optimization problems [41]. In this work, a Helmholtz-type PDE-filter [42] is applied since it can be easily implemented in the utilized FEM software and allows for computationally efficient filtering. The filter PDE is stated by:

$-r_{\text {filter }}^{2}\left(\frac{\partial^{2} \tilde{\gamma}}{\partial x^{2}}+\frac{\partial^{2} \tilde{\gamma}}{\partial y^{2}}\right)+\tilde{\gamma}=\gamma$

where $r_{\text {filter }}$ is the filter parameter and $\tilde{\gamma}$ is the filtered design variable. A symmetry boundary condition is applied at the left, right, and bottom of the design domain and $\tilde{\gamma}$ is prescribed to be solid at the boundary to the wall. This leads to the following set of boundary conditions:

$-\mathbf{n} \cdot(-\nabla \tilde{\gamma})=0 \quad$ on $\Gamma_{d s}$

$\tilde{\gamma}=0 \quad$ on $\Gamma_{w d}$

The density filter inherently introduces a band of intermediate densities between the solid and fluid region which is physically not meaningful and adds an artificial heat transfer resistance between the solid and fluid domains in 
this specific modeling approach ${ }^{2}$. To obtain a sharper solid-fluid transition, a smooth Heaviside projection [43] is used, which is given by:

$\overline{\tilde{\gamma}}=\frac{\tanh (\beta \eta)+\tanh (\beta(\tilde{\gamma}-\eta)}{\tanh (\beta \eta)+\tanh (\beta(1-\eta))}$

where $\overline{\tilde{\gamma}}$ is the projected design variable, $\eta$ is a threshold parameter and $\beta$ determines the steepness of the projection. The reader should note that $\overline{\tilde{\gamma}}$ becomes the physically meaningful design variable in the interpolations stated in section 2 .

\subsection{Computational implementation}

The topology optimization model is implemented in the commercial simulation software COMSOL Multiphysics [44]. COMSOL's heat transfer module is used to solve the energy equation (Eq. (8)). The Navier-Stokes equation and filter PDE are implemented in COMSOL's coefficient form PDE interface in which the PDEs can be stated as indicated in Eq. (3) and Eq. (14). A first order discretization is used to solve the state equations of the thermofluid problem and the filter PDE. The optimization is conducted with COMSOL's optimization module using the globally convergent version of the Method of Moving Asymptotes (GCMMA) [45] where the constraint for the air bulk temperature is implemented as a global constraint. The adjoint problem is solved automatically in COMSOL to obtain the sensitivities for the objective and constraint functional. COMSOL is interfaced with MATLAB to allow for automatized parameter changes during the optimization. Changing parameter values during the optimization is necessary because a continuation approach [41] is applied to the convexity parameter $b_{k}$ of the conductivity interpolation. This is done to ensure a relatively convex optimization problem at the beginning of the process and to increasingly penalize the thermal conductivity of intermediate densities. The sequence of values $b_{k}=\{0,2,10,10\}$ is used for all optimizations presented in this study. During the ramping of $b_{k}$, the projection steepness parameter $\beta$ is held constant at a value of 5 in order to reduce the grey band between solid and fluid areas. After ramping $b_{k}, \beta$ is set to a value of 10 to obtain final designs with a sharp solid-fluid transition which correspond to the sequence of values $\beta=\{5,5,5,10\}$. The projection threshold parameter $\eta$ is held at a constant value of 0.5 throughout the optimization and an initial design variable density of 0.5 is set in the entire design domain in all optimizations. Other model parameters that are kept constant during the optimization are stated in Table 1.

\footnotetext{
${ }^{2}$ The artificial heat transfer resistance occurs because the fluid flow is already penalized and close to zero in the grey area due to a relatively high maximum friction force. At the same time is the thermal conductivity in the grey area significantly lower than pure filled polymer; especially with increasing convexity of the conductivity interpolation.
} 
Table 1. Model and optimization parameter values.

\begin{tabular}{ll}
\hline Parameter & Value \\
\hline $\bar{\alpha}$ & $2 \times 10^{5} \mathrm{~Pa} \mathrm{~s} \mathrm{~m}^{-2}$ \\
Design domain width & $4 \mathrm{~mm}$ \\
Maximum element size in mesh & $3.25 \times 10^{-5} \mathrm{~m}$ \\
$r_{\text {filter }}$ & $4.3 \times 10^{-5} \mathrm{~m}$ \\
\hline
\end{tabular}

\section{Results}

The topology optimization model is used to generate optimized structures for different unit cell heights and effective composite conductivities. Air channel heights between $2 \mathrm{~mm}$ and $14 \mathrm{~mm}$ are considered, where the thickness of the wall between the air and the water channel is held constant at $1.5 \mathrm{~mm}$. The thermal conductivities are varied between $0.1 \mathrm{~W} /(\mathrm{m} \mathrm{K})$, which is in the range of unfilled plastics such as acrylonitrile butadiene styrene (ABS), and $300 \mathrm{~W} /(\mathrm{m} \mathrm{K})$ which is on the order of metallic heat sink materials. The parameters defining the operating conditions of the heat exchanger are selected to be similar to actual operating conditions anticipated in dry-cooled power plant condensers [46]. Heat capacity, thermal conductivity, viscosity, and density of air are considered constant in this work and are evaluated at a temperature of $45^{\circ} \mathrm{C}$. The values of the constant, unless explicitly stated otherwise, heat exchanger operating parameters and thermophysical properties of air used in the parameter studies are given in Table 2.

Table 2. Values of the constant, unless explicitly stated otherwise, heat exchanger operating parameters and thermophysical properties of air used in this work.

\begin{tabular}{llll}
\hline Operating parameter & Value & Thermophysical properties of air & Value \\
\hline$\frac{\Delta p_{H E X}}{L}$ & $4000[\mathrm{~Pa} / \mathrm{m}]$ & $c_{\text {air }}$ & $1006[\mathrm{~J} /(\mathrm{kg} \mathrm{K})]$ \\
$\frac{\Delta T_{H E X}}{L}$ & $1200[\mathrm{~K} / \mathrm{m}]$ & $k_{\text {air }}$ & $0.027[\mathrm{~W} /(\mathrm{m} \mathrm{K})]$ \\
$T_{\text {water }}$ & $70\left[{ }^{\circ} \mathrm{C}\right]$ & $\mu_{\text {air }}$ & $1.941 \times 10^{-5}[\mathrm{~Pa} \mathrm{~s}]$ \\
$T_{\text {air, bulk }}^{*}$ & $45\left[{ }^{\circ} \mathrm{C}\right]$ & $\rho_{\text {air }}$ & $1.112\left[\mathrm{~kg} / \mathrm{m}^{3}\right]$ \\
\hline
\end{tabular}

An optimized design and the corresponding fluid velocity field and temperature distribution is shown in Fig. 3 for an air channel height of $6 \mathrm{~mm}$ and thermal conductivity of $5 \mathrm{~W} /(\mathrm{m} \mathrm{K})$ in order to illustrate a typical result. The optimized design consists of three fins connected to the wall separating water and air flow. The three fins are roughly of the same size and have smaller secondary branches. The fluid channels tend to become slightly larger closer to the top boundary of the design domain. Consequently, the fluid flow is increasing closer to the hot water boundary which allows heat transfer between the (not explicitly modeled) water and air flow to take place with minimal heat transfer resistance in the polymer. The temperature distribution shown in Fig. 3 (c) shows the cool air channels in the design and the warmer heat exchanger material. It can be seen how the dendritic structures conduct the heat relatively evenly in all parts of the design domain. The Reynolds number of the air flow in the optimized design is of interest to verify the laminar flow assumption that was used for the 
thermofluid modeling. However, this Reynolds number cannot be determined in a straightforward way due to the unconventional channel geometry. For this reason, it is estimated based on a pipe flow and flow between parallel plates assumption. The computation of the air flow Reynolds number of the design from Fig. 3 and another exemplary design is described in Appendix A. The Reynolds number of the design from Fig. 3 is 271 assuming pipe flow and 318 assuming flow between parallel plates which is significantly lower than the critical Reynolds number for laminar-turbulent transition which is for pipe flow reported to be between 2040 [47] and 2100 [48] and for flow between parallel plates slightly below 2300 [48]. Similar results with the estimated air flow Reynolds number being well below the critical Reynolds number are obtained for all designs generated in this work with maximal values of around 650 which justifies the laminar flow assumption that was used in the presented modeling approach. Nevertheless, thermofluid topology optimization including a turbulence closure model could result in different optimized geometries than the designs obtained in this study due to the differences in the heat transfer modeling. For example broader air channels in which turbulent flow occurs might be formed. However, including a turbulence closure model in the topology optimization and benchmarking the results against those of the current study will be left for future works as research on turbulent flow topology optimization is still in its very beginnings.

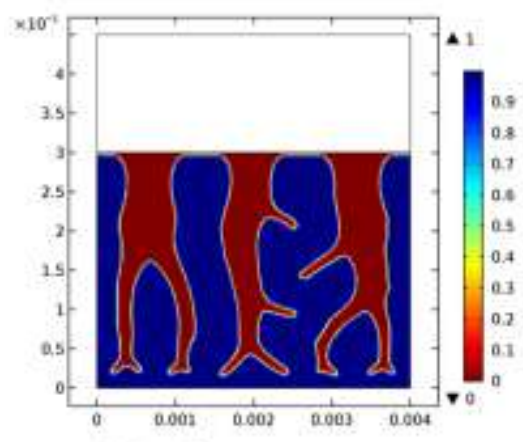

(a) Design

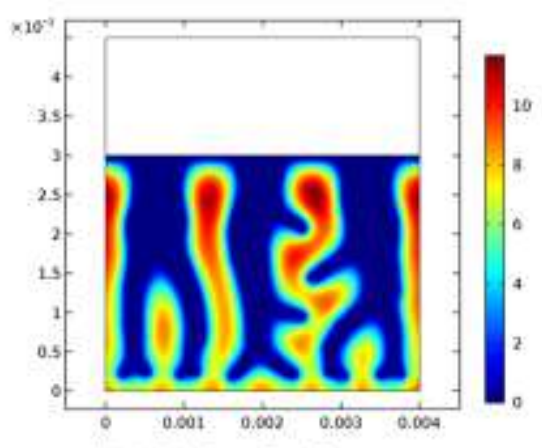

(b) Velocity $[\mathrm{m} / \mathrm{s}]$

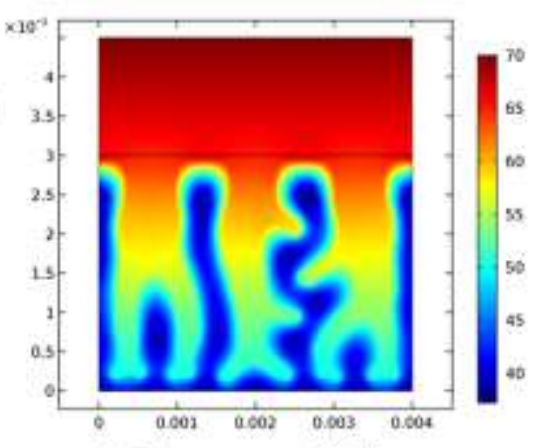

(c) Temperature $\left[{ }^{\circ} \mathrm{C}\right]$

Fig. 3. Optimized design for $5 \mathrm{~W} /(\mathrm{m} \mathrm{K})$ and an air channel height of $6 \mathrm{~mm}$. (a) shows the geometry (red is solid, blue is air, and white is the wall between the air and water passages, which is not part of the optimization design domain) as well as the corresponding velocity field (b) and temperature distribution (c).

\subsection{Parametric study of system performance}

Optimized designs for a composite thermal conductivity of $5 \mathrm{~W} /(\mathrm{m} \mathrm{K})$ with several different air channel/unit cell heights are depicted in Fig. 4. For an air channel height of $2 \mathrm{~mm}$, four small fins evolve whereas for the larger air channel heights three fins evolve in each case. Again, the dendritic shape of the fins and slightly larger fluid channels close to the top boundary of the design domain can be seen. Furthermore, it is interesting to note that in the $20 \mathrm{~mm}$ air channel height design, the fluid channels are placed only in the top $75 \%$ of the design domain and the bottom region is filled entirely with solid. This shows that the composite conductivity is, for this unit cell height, not high enough to make it attractive to distribute the air channels within the entire design domain. There is wasted polymer material placed in the regions far from the water just to prevent air from flowing so far from 
the water. Slight grey areas occur in this design at the transition from the dendritic fin to the polymer material without fluid channels.

Fig. 5 shows optimized designs for an air channel height of $6 \mathrm{~mm}$ for different effective composite thermal conductivities. Design (a), which corresponds to a conductivity of $0.1 \mathrm{~W} /(\mathrm{m} \mathrm{K})$, has only one horizontal fluid channel directly next to the wall that separates the air flow from the water. The rest of the design domain is filled with polymer to prevent air flow. Design (b) consists of four fins: two smaller fins in the middle and a larger dendritic one at each side of the design domain. The larger fins are quite thick in order to prevent too much air flow at the bottom of the design domain. The designs (c), (d), and (e) consist each of three fins with secondary branches from the main fin. An increasingly finer feature size can be observed with increasing thermal conductivity. The fact that smaller feature sizes become increasingly advantageous leads to grey regions in the secondary branches in design (f) and to a smaller extent in design (e). However, a black and white design could be obtained using a finer mesh and smaller filter radius allowing a finer design resolution or by implementing robust topology optimization approaches $[43,49]$ that guarantee a minimum length scale in the solid which is left for future works. Similar findings regarding the design's conductivity dependence are obtained for other unit cell heights. It is interesting to observe that design (c) is nearly symmetric with respect to a vertical line in the center of the modeled domain which is not enforced by the boundary conditions that only enforce symmetry with respect to the left, right, and bottom boundary of the design domain. This additional symmetry line in the center of the modeled domain seems to be advantageous from a design point of view in this specific case; however, the other optimized designs are asymmetric with regards to this line.

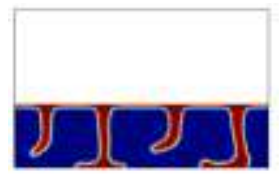

(a)

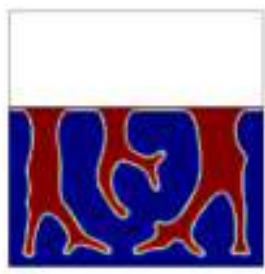

(b)

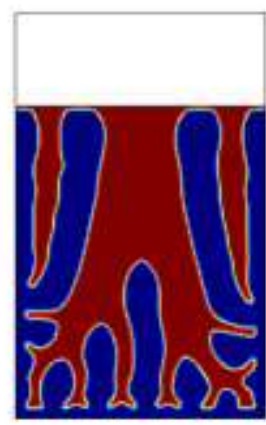

(c)

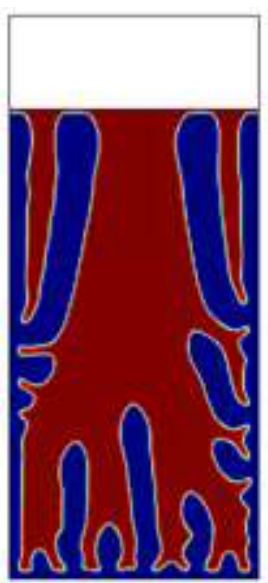

(d)

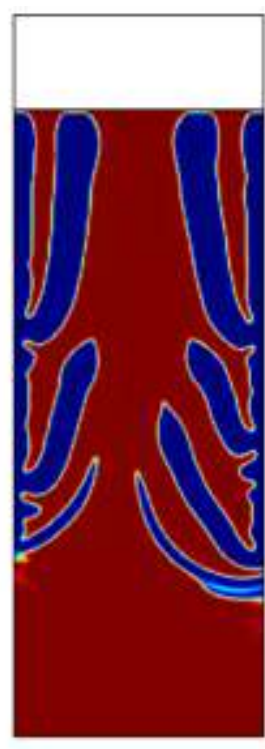

(e)

Fig. 4. Optimized designs for $5 \mathrm{~W} /(\mathrm{m} \mathrm{K})$. The air channel height is taking the values of $2 \mathrm{~mm}(\mathrm{a}), 5 \mathrm{~mm}(\mathrm{~b}), 10 \mathrm{~mm}(\mathrm{c}), 15 \mathrm{~mm}(\mathrm{~d})$, and $20 \mathrm{~mm}$ (e). Red corresponds to polymer and blue to fluid passages. White is the unit cell wall (polymer) which is not part of the design domain. 


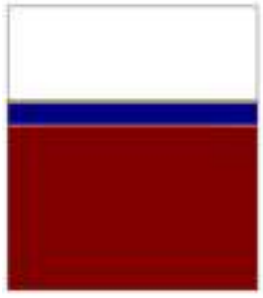

(a)

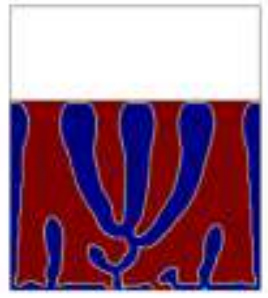

(b)

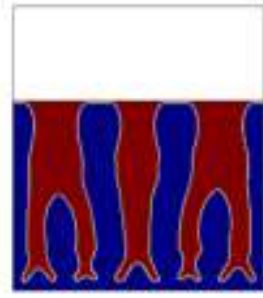

(c)

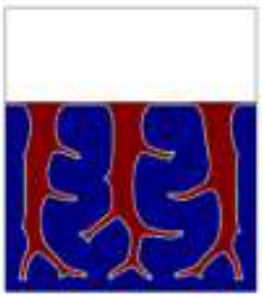

(d)

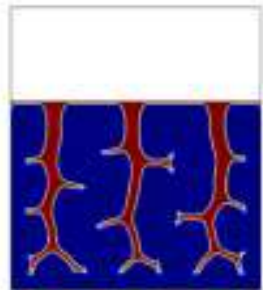

(e)

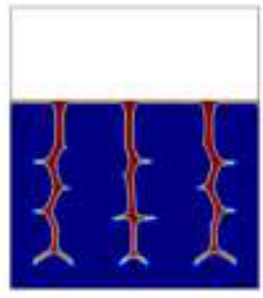

(f)

Fig. 5. Optimized designs for different conductivities for an air channel height of $6 \mathrm{~mm}$. The polymer composite conductivity is increasing from left to right taking the values of 0.1 (a), 0.5 (b), 2 (c), 10 (d), 50 (e), and 300 (f) W/(m K) respectively. Red corresponds to polymer and blue to fluid. White is the unit cell wall (polymer) which is not part of the optimizable domain.

As stated above, the operating parameters pressure drop over heat exchanger flow length and temperature drop over heat exchanger flow length are selected to be consistent with a specific application in this work. Still, it is interesting to examine the effect of varying these parameters on the optimization and the resulting optimized designs. Fig. 6 shows the effect of either halving or doubling one of these operating parameters while keeping the other parameter at the reference value for a constant air channel height of $4 \mathrm{~mm}$ and effective composite conductivity of $5 \mathrm{~W} /(\mathrm{m} \mathrm{K})$. Design (a) is optimized for the reference values of pressure drop and temperature increase over the heat exchanger which are used throughout this work and stated in Table 2. Two larger fins at the side of the design domain with secondary and small tertiary branches and a smaller fin in the middle of the design domain are generated. Halving either the pressure drop or the temperature increase over the heat exchanger results in relatively similar designs ((b) and (c)). However, the air channels between the fins are slightly larger in these designs compared to design (a) which is due to the lower pressure drop (b) or lower axial temperature increase (c). Doubling either the pressure drop or temperature increase over the heat exchanger results in designs with four main fins ((d) and (e)) and significantly smaller air channels between the fins as in the other cases. This is due to the fact that more heat can be transported away by the air flow per unit flow area due to higher flow velocities (increased pressure drop) or higher axial temperature gradient (increased temperature increase over the heat exchanger).

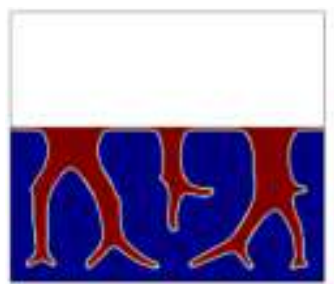

(a)

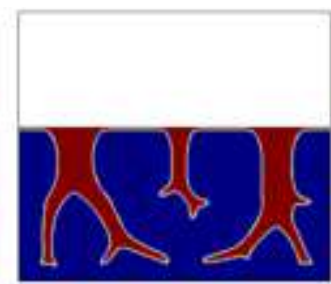

(b)

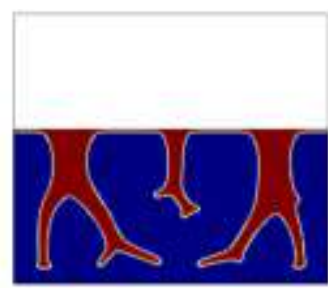

(c)

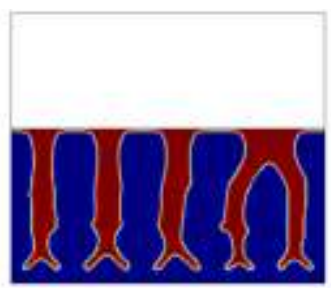

(d)

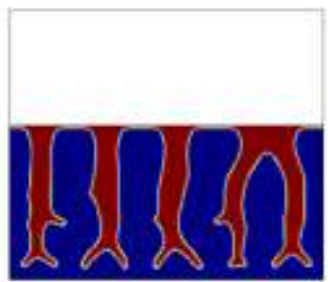

(e)

Fig. 6. Influence of the operating parameters pressure drop over heat exchanger flow length and temperature increase over heat exchanger flow length on the optimized designs for $5 \mathrm{~W} /(\mathrm{m} \mathrm{K})$ and an air channel height of $4 \mathrm{~mm}$. Design (a) corresponds to the standard case of a $4000 \mathrm{~Pa} / \mathrm{m}$ pressure drop and $1200 \mathrm{~K} / \mathrm{m}$ temperature increase. In the other cases the pressure drop and temperature increase take the values of $2000 \mathrm{~Pa} / \mathrm{m}$ and $1200 \mathrm{~K} / \mathrm{m}$ (b), $4000 \mathrm{~Pa} / \mathrm{m}$ and $600 \mathrm{~K} / \mathrm{m}$ (c), $8000 \mathrm{~Pa} / \mathrm{m}$ and $1200 \mathrm{~K} / \mathrm{m} \mathrm{(d),} 4000 \mathrm{~Pa} / \mathrm{m} \mathrm{and} 2400 \mathrm{~K} / \mathrm{m}$ (e), respectively. 


\section{$\underline{\text { Parameter studies }}$}

In the subsequent section, the influence of the composite thermal conductivity on the optimal air channel height will be discussed for different measures of the heat exchanger's performance. Fig. 7 shows the heat exchanger conductance per volume (total volume of polymer composite, air, and water channels) plotted against the air channel height for different polymer thermal conductivities. To calculate the heat exchanger volume, a constant water channel height of $1 \mathrm{~mm}$ is assumed to exist between the unit cells. It can be seen that both the effective thermal conductivity and the air channel height have a significant influence on the system performance. The optimal air channel height increases with increasing thermal conductivity. For conductivities of $0.1,0.5$, and 2 $\mathrm{W} /(\mathrm{m} \mathrm{K})$, a $2 \mathrm{~mm}$ air channel height (the smallest value considered) is found optimal. The optimal air channel height increases to approximately 4, 6, 8, and $12 \mathrm{~mm}$ for the thermal conductivities of 5, 10, 50, and $300 \mathrm{~W} /(\mathrm{m}$ $\mathrm{K})$ respectively. The increase of the optimal air channel height with increasing thermal conductivity can be explained by the reduced thermal resistance in the heat transfer enhancing structures that can therefore productively extend further away from the water cooled wall.

Another metric that can be used to examine the heat exchanger performance is conductance per unit heat exchanger mass since the amount of polymer needed significantly influences in additive manufacturing the manufacturing time and cost. The heat exchanger conductance per polymer mass vs. the air channel height is depicted for different thermal conductivities in Fig. 8. For these studies, a composite density of $1000 \mathrm{~kg} / \mathrm{m}^{3}$ which corresponds to the density of ABS is assumed. It should be noted that this is an approximation for higher effective thermal conductivities of the composite as the conductive filler density can differ from the polymer density. The optimal air channel heights, when considering conductance per mass, behave similarly to conductance per volume. For conductivities of 0.1 and $0.5 \mathrm{~W} /(\mathrm{m} \mathrm{K})$ an air channel height of $2 \mathrm{~mm}$ is optimal. For conductivities of 2, 5, 10, 50, and $300 \mathrm{~W} /(\mathrm{m} \mathrm{K})$ the optimal heights are approximately 4, 4, 6, 10, and 12 $\mathrm{mm}$, respectively. For lower thermal conductivities, the dependence of conductance per mass on the air channel height is less pronounced than when considering conductance per unit volume. 


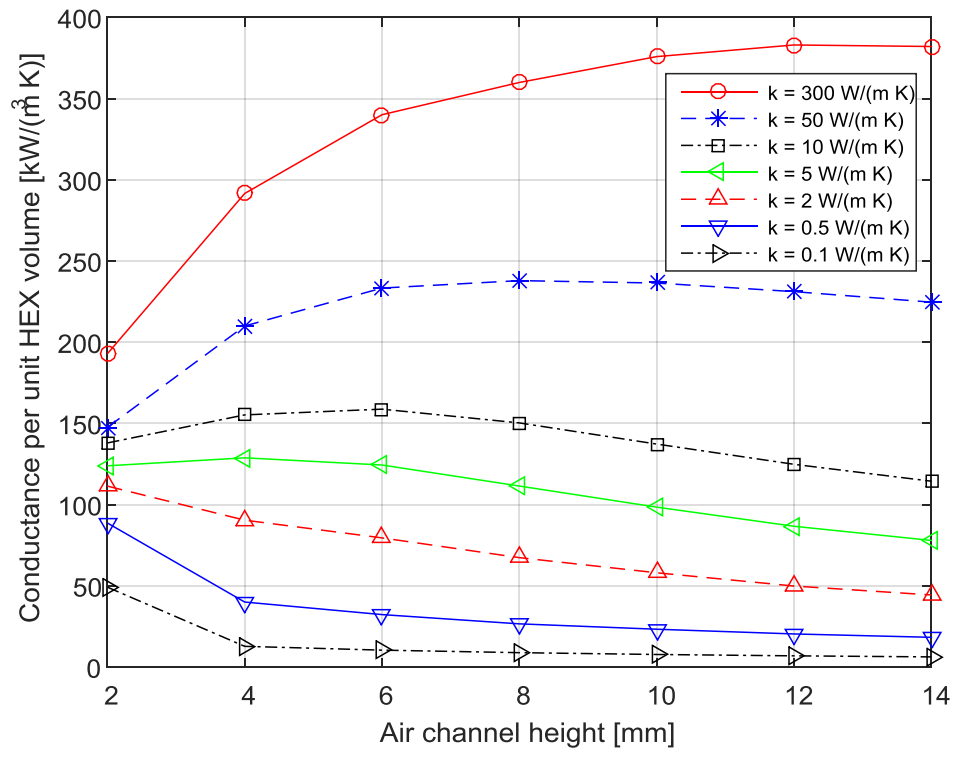

Fig. 7. Heat exchanger's conductance per heat exchanger unit volume plotted against the air channel height for different thermal conductivities of the polymer.

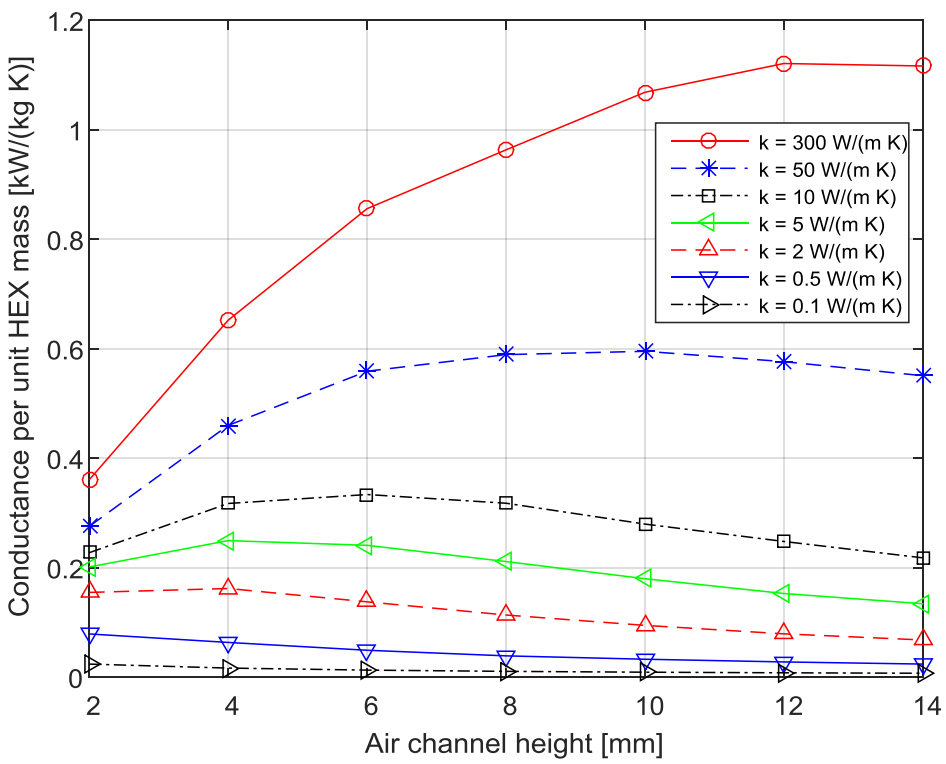

Fig. 8. Heat exchanger's conductance per unit heat exchanger mass plotted against the air channel height for different values of thermal conductivity.

The effect of varying the operating parameters pressure drop over heat exchanger flow length and temperature increase over heat exchanger flow length on the heat exchanger conductance per volume and conductance per mass for a constant effective composite conductivity of $5 \mathrm{~W} /(\mathrm{m} \mathrm{K})$ and constant air channel height of $4 \mathrm{~mm}$ is shown in Table 3. Halving the pressure drop results in a decrease of heat exchanger conductance per volume and 
conductance per mass of around 18\% compared to the reference case. Doubling the pressure drop yields a conductance per volume increase of $25 \%$ and a conductance per mass increase of $20 \%$. Varying the temperature increase over the heat exchanger has a similar effect, halving the axial temperature increase results in a conductance decrease of $23 \%$ on a per volume basis and $19 \%$ on a per mass basis. Doubling the axial temperature increase yields a conductance per volume increase of $30 \%$ and a conductance per mass increase of $25 \%$.

Table 3. Effect of varying the operating parameters pressure drop over heat exchanger flow length and temperature increase over heat exchanger flow length on the heat exchanger conductance per unit volume and conductance per unit mass. The effective composite conductivity is $5 \mathrm{~W} /(\mathrm{m} \mathrm{K})$ and the air channel height is $4 \mathrm{~mm}$.

\begin{tabular}{llllc}
\hline $\begin{array}{l}\text { Design in } \\
\text { Fig. 6 }\end{array}$ & $\frac{\Delta p_{H E X}}{L}[\mathrm{~Pa} / \mathrm{m}]$ & $\frac{\Delta T_{H E X}}{L}[\mathrm{~Pa} / \mathrm{m}]$ & $\begin{array}{l}\text { Conductance/unit HEX volume } \\
{\left[\mathrm{kW} /\left(\mathrm{m}^{3} \mathrm{~K}\right)\right]}\end{array}$ & $\begin{array}{l}\text { Conductance/unit HEX mass } \\
{[\mathrm{kW} /(\mathrm{kg} \mathrm{K})]}\end{array}$ \\
\hline (a) & 4000 & 1200 & 128.9 & 0.249 \\
(b) & 2000 & 1200 & 103.4 & 0.208 \\
(c) & 4000 & 600 & 99.7 & 0.201 \\
(d) & 8000 & 1200 & 160.8 & 0.299 \\
(e) & 4000 & 2400 & 167.4 & 0.312 \\
\hline
\end{tabular}

It should be kept in mind that the presented studies are conducted using the assumption of a 2D fully developed flow to obtain a stable and not too computationally expensive optimization model as stated in chapter 2 . Even though assuming a fully developed flow is a mature technique in thermofluid modeling, an experimental validation of the designs' performance would be beneficial to quantify the influence of the assumptions that are made. This is left for a future work as this paper focuses on the theoretical aspects of the fully developed flow model and its application to the presented specific heat exchanger design problem. To assess the influence of the mesh resolution on the modeling of the thermofluid problem, three representative optimized designs $(0.5 \mathrm{~W} /(\mathrm{m}$ $\mathrm{K})$ and $2 \mathrm{~mm}$ air channel height; $5 \mathrm{~W} /(\mathrm{m} \mathrm{K})$ and $4 \mathrm{~mm} ; 10 \mathrm{~W} /(\mathrm{m} \mathrm{K})$ and $6 \mathrm{~mm})$ are re-evaluated using a maximum element size in the mesh of $50 \%$ and $25 \%$ of the value used throughout this work which is stated in Table 1. The maximum deviation between the meshes in terms of predicted conductance per volume is found to be $2.17 \%$ and $2.16 \%$ in terms of conductance per mass for the case $0.5 \mathrm{~W} /(\mathrm{m} \mathrm{K})$ and air channel height of $2 \mathrm{~mm}$. The maximum conductance deviation between the meshes for the other two optimized designs is around $1.5 \%$ both on a per volume basis and per mass basis. This suggests that the accuracy of the computations can still be slightly increased when using a finer mesh than the one used in this work which is chosen as a trade-off between accuracy and computational demand. Nevertheless, the uncertainty due to the mesh resolution seems acceptable compared to the uncertainty that is associated with the assumptions on which the presented optimization model relies.

To provide some validation of the fluid-side fully developed flow thermofluid model used in this work, a comparison to analytical solutions for fully developed flow Nusselt numbers in simple geometries, i.e. flow between parallel plates and flow in a circular pipe, is conducted. The 2D thermofluid problem in these geometries is modeled with a prescribed temperature at the boundary to the, in this validation model not explicitly considered, solid, while constraining the difference between the prescribed temperature at the solid 
boundary $\left(T_{\text {wall }}\right)$ and the air bulk temperature. This corresponds to a 3D problem with in axial (or z) direction constant heat flux from the wall to the duct as the mass flow in the duct, the fluid properties, the duct geometry, and the driving force for the heat transfer $\left(T_{\text {wall }}(z)-T_{\text {air,bulk }}(z)\right)$ are constant along the axial coordinate. Hence, the Nusselt number obtained from this numerical model can be compared to analytical solutions for fully developed flow Nusselt numbers for constant heat flux from the wall in axial direction which are stated in [50] for flow between parallel plates and in [51] for flow in a circular tube. A relative deviation between numerical model and correlations of around $0.1 \%$ was found for the same maximum element size in the mesh as used in the optimization model which confirms the validity of the utilized modeling approach. The calculations done for this comparison are presented in Appendix B. It is interesting to note that the deviation of numerical validation model and analytical solution in terms of Nusselt number is lower than the deviation of conductance in the optimized geometries when refining the mesh. This is probably due to the simpler geometries used in the validation model compared to the topology optimized geometries.

It is important to state that the chosen modeling approach provides a conservative estimate for the heat transfer in the geometries as the local heat transfer in the entrance region of a duct or similar geometry is always higher than in the fully developed region [52]. A comparison to experiments done within the ARPA-E ARID project [53] within which also this study was conducted indicates an increased performance of the topology optimized geometries compared to conventionally designed 3D-printed heat exchangers in terms of conductance per heat exchanger mass. However, it is not possible to reliably quantify this improvement based on the currently available experimental data. For this reason, it was decided to consistently compare the topology optimized designs to designs generated by a simpler optimization model in this study. This benchmarking to size optimized slot channel designs that represent a simpler but established heat exchanger geometry is presented in the following section.

\subsection{Comparison to size optimized slot channel model}

To validate the improved performance of the topology optimized designs, the results are compared to a model that is mathematically similar to the topology optimization model but consists of an arbitrary number of vertical slots for air flow. The slot width of this model is adjusted to maximize the conductance per unit heat exchanger volume for a given number of slots subject to the same bulk temperature constraint that is applied to the topology optimization model. The width of the modeled domain is $4 \mathrm{~mm}$ as it is when conducting the topology optimization. This allows for a fair comparison between a conventional, simple heat exchanger geometry that is optimized in a straightforward way and the less intuitive and more complex structures that are obtained using the topology optimization approach. Symmetry boundary conditions are applied at the left, right, and bottom boundaries of the modeled domain as in the topology optimization model to obtain results which are representative of the entire unit cell. The slot width is optimized for air channel heights of 2, 5, and $10 \mathrm{~mm}$ and thermal conductivities of $0.5,2,5,10,50$, and $300 \mathrm{~W} /(\mathrm{m} \mathrm{K})$. For each conductivity, an optimal number of slots and optimal air channel height is determined. For example, for a conductivity of $5 \mathrm{~W} /(\mathrm{m} \mathrm{K})$, 5 fins with a width of $0.24 \mathrm{~mm}$ and an air channel height of $5 \mathrm{~mm}$ are found to be optimal. The resulting design and the corresponding air velocity and temperature distribution are depicted in Fig. 9. A summary of the optimal air channel height, optimal number of fins, and optimal fin width for all thermal conductivities is given in Table 3. 


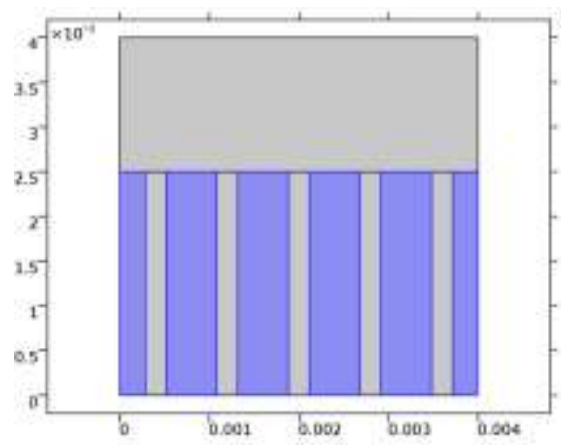

(a) Design

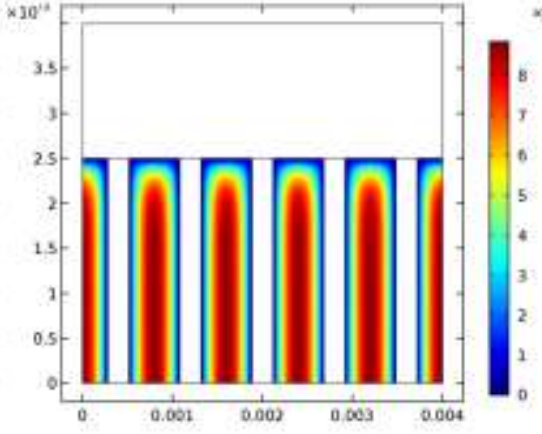

(b) Velocity $[\mathrm{m} / \mathrm{s}]$

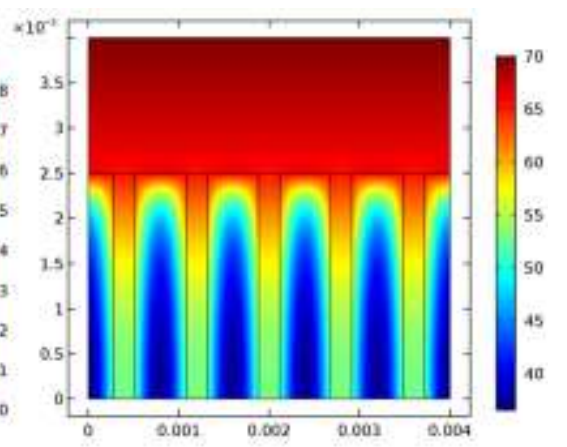

(c) Temperature $\left[{ }^{\circ} \mathrm{C}\right]$

Fig. 9. The optimal slot channel model design for $5 \mathrm{~W} /(\mathrm{m} \mathrm{K})$ (a) where the fins are depicted in grey and fluid slots are shown in blue as well as the corresponding velocity field (b) and temperature distribution (c).

Table 3. Optimal air channel height, optimal number of fins, and optimal fin width for the analyzed composite effective thermal conductivities in the slot channel model.

\begin{tabular}{llll}
\hline Conductivity $[\mathrm{W} /(\mathrm{m} \mathrm{K})]$ & $\begin{array}{l}\text { Optimal air channel height } \\
{[\mathrm{mm}]}\end{array}$ & Optimal number of fins & $\begin{array}{l}\text { Optimal fin width } \\
{[\mathrm{mm}]}\end{array}$ \\
\hline 0.5 & 2 & 5 & 0.31 \\
2 & 2 & 5 & 0.17 \\
5 & 5 & 5 & 0.25 \\
10 & 5 & 5 & 0.19 \\
50 & 10 & 6 & 0.08 \\
300 & 10 & 6 & 0.03 \\
\hline
\end{tabular}

The slot channel designs with optimal number of slots, slot width and air channel height for the respective conductivity are shown in Fig. 10. The topology optimized designs for each thermal conductivity at the optimal height (out of 2,5, and $10 \mathrm{~mm}$ air channel heights) are added to the figure to allow a qualitative comparison between the two optimization techniques. It can be seen that the optimal air channel height is approximately the same in the slot channel and topology optimization model for the same conductivity: for conductivities of 0.5 and $2 \mathrm{~W} /(\mathrm{m} \mathrm{K})$ a $2 \mathrm{~mm}$ height is optimal, for 5 and $10 \mathrm{~W} /(\mathrm{m} \mathrm{K})$ a $5 \mathrm{~mm}$ height is optimal, and for 50 and 300 $\mathrm{W} /(\mathrm{m} \mathrm{K})$ a $10 \mathrm{~mm}$ air channel height yields the highest conductance. The largest optimal fin width is $0.31 \mathrm{~mm}$ for $0.5 \mathrm{~W} /(\mathrm{m} \mathrm{K})$ and the smallest optimal fin width is $0.03 \mathrm{~mm}$ and occurs for $300 \mathrm{~W} /(\mathrm{m} \mathrm{K})$. Five fins are optimal for all slot channel model designs except for conductivities of 50 and $300 \mathrm{~W} /(\mathrm{m} \mathrm{K})$ where six fins are optimal. Compared to this, fewer, thicker fins are generated by the topology optimization: Four fins occur for 0.5 and $2 \mathrm{~W} /(\mathrm{m} \mathrm{K})$, three fins for 5 and $10 \mathrm{~W} /(\mathrm{m} \mathrm{K})$ and 2 fins for 50 and $300 \mathrm{~W} /(\mathrm{m} \mathrm{K})$. These fins, however, have finer secondary and in some cases tertiary branches that conduct the heat evenly in the air flow; hence allowing for a lower total number of fins. These thicker fins with finer branches are more suited for additive manufacturing, if necessary with minor post processing after the topology optimization, than the very thin fins in the slot channel model that occur for higher composite conductivities. As for the topology optimized designs, the air flow Reynolds number in the slot channel model is determined to verify the laminar flow assumption. This is 
straightforward since the flow in each slot can be considered as a flow between two parallel plates. The highest Reynolds number is obtained for the design in Fig. 10 (f). The determined value of 257 is significantly below the critical Reynolds number for laminar-turbulent transition in flow between two parallel plates that is reported to be slightly below 2300 [48] which justifies the laminar flow assumption used in the slot channel model. The air bulk velocity and characteristic length used to determine the Reynolds number of the slot channel design from Fig. 10 (f) are stated in Table A.1 in Appendix A.
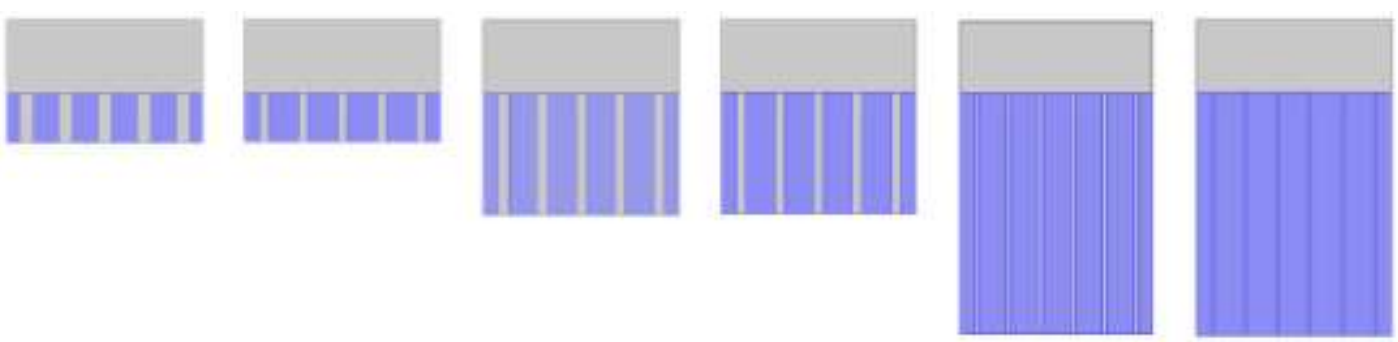

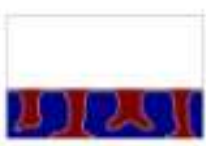

(a)

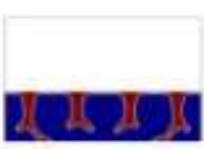

(b)

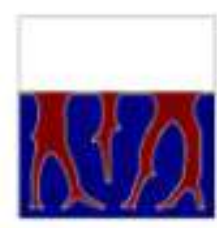

(c)

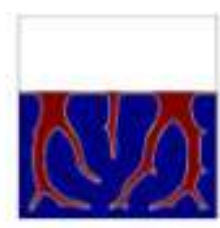

(d)

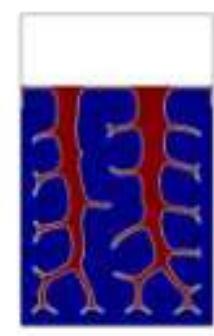

(c)

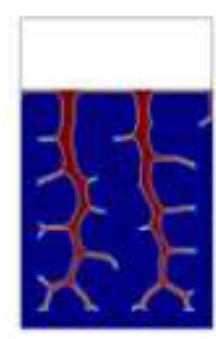

(f)

Fig. 10. Slot channel designs with optimal air channel height, number of fins, and fin width for thermal conductivities of 0.5 (a), 2 (b), 5 (c), 10 (d), 50 (e), and 300 (f) W/(m K). Fins and wall to water are depicted in grey and air channels in blue. The topology optimized designs for the same respective conductivity are added for comparison below where red corresponds to fins and blue to void passages for air flow.

To assess the manufacturability of the topology optimization and slot channel model designs in a more quantitative way, the aspect ratio, i.e. length over width of fins, is compared. However, defining the aspect ratio in the topology optimized designs is not straightforward since the fins in the same design have different lengths and widths and the fin width might vary along the length. Moreover, there are thinner secondary branches which are each characterized by their own aspect ratio. To still be able to approximatively assess the aspect ratio in the topology optimized designs, the following procedure was conducted: For each fin in the respective design, the aspect ratio is determined for the main structure of the fin using an average width. Shorter secondary branches are not considered since they are much shorter than the main fin, thus having a lower aspect ratio even though their width is smaller than the width of the main branch. Then for each design the maximum aspect ratio among all fins is chosen as representative aspect ratio for the entire design. This aspect ratio of the topology optimized designs and the aspect ratio of the slot channel plotted against the effective composite conductivity are shown in Fig. 11. In addition, the aspect ratio of a slot channel model having a minimum fin width constraint of $0.3 \mathrm{~mm}$ is also shown in the figure for comparison. 


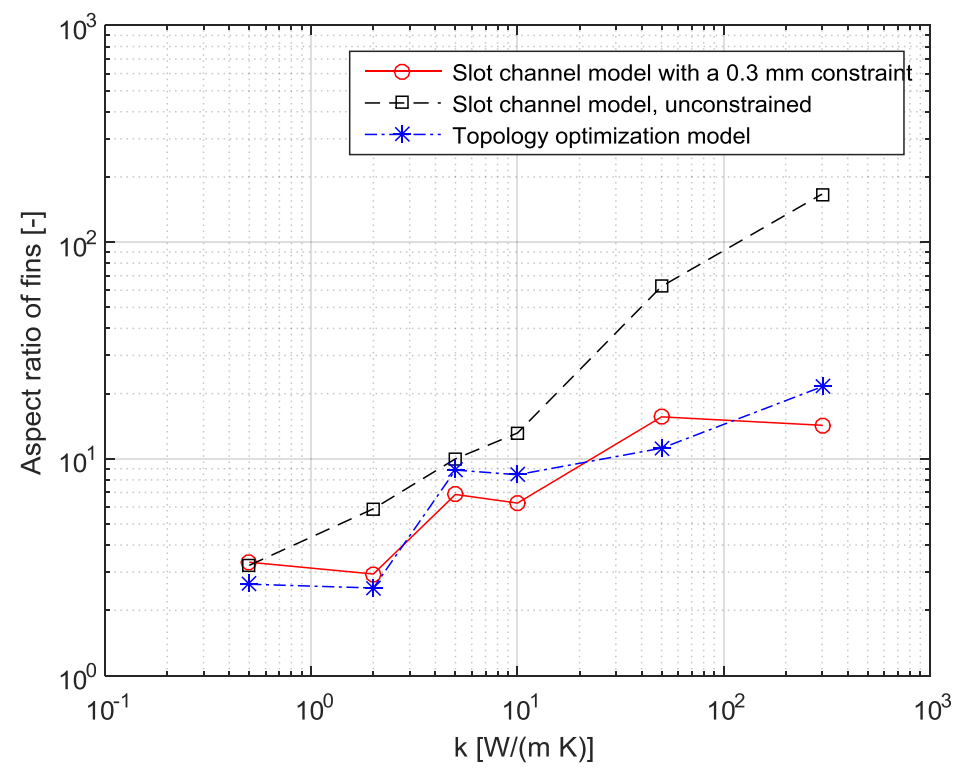

Fig. 11. Aspect ratio defined as fin length over fin width of the slot channel model with a $0.3 \mathrm{~mm}$ minimum fin width constraint, of the unconstrained slot channel model, and of the topology optimization model.

It can be seen that the aspect ratio of the topology optimized designs and the fin width constraint slot channel model are on the same order for all thermal conductivities. The aspect ratio of the unconstrained slot channel model is higher than the value associated with the other two models for all thermal conductivities except for 0.5 $\mathrm{W} /(\mathrm{m} \mathrm{K})$. However, for lower conductivities the aspect ratio is still in the same order of magnitude - only for 50 and $300 \mathrm{~W} /(\mathrm{m} \mathrm{K})$ is the aspect ratio of the unconstrained model much larger (around one order of magnitude higher) than for the constrained slot channel and the topology optimization model. Observing the trend of the aspect ratio with increasing conductivity, one can see that the aspect ratio of the unconstrained slot channel model is continuously increasing with increasing conductivity. Compared to this, the constrained slot channel model and the topology optimization model also show a general trend of increasing aspect ratio with increasing conductivity but have relatively similar aspect ratios for the same optimal air channel heights and significant increase of the aspect ratio when the optimal air channel height changes from 2 to $5 \mathrm{~mm}$ and from 5 to $10 \mathrm{~mm}$ which corresponds to conductivities changing from 2 to $5 \mathrm{~W} /(\mathrm{m} \mathrm{K})$ and from 10 to $50 \mathrm{~W} /(\mathrm{m} \mathrm{K})$.

A quantitative comparison between the performance of the slot channel designs and the topology optimized designs can be seen in Fig. 12, where the improvement in conductance per unit heat exchanger volume of the topology optimized designs compared to the slot channel model is plotted against the composite effective thermal conductivity. This comparison is conducted with no constraint on the fin width and a $0.3 \mathrm{~mm}$ minimum fin width constraint in the slot channel model. However, it should be noted that comparing the width constrained slot channel model to the topology optimized model is not entirely fair since no robust length scale control is implemented in the topology optimization even though the density filter mostly prevents very thin features from appearing in the final designs as can be seen in Fig. 10. Fig. 12 shows that for $0.5 \mathrm{~W} /(\mathrm{m} \mathrm{K})$ the constrained and unconstrained slot channel model yield the same result relative to the topology optimization model since the optimal fin width in the slot channel model is $0.31 \mathrm{~mm}$. For higher conductivities, the optimal fin width in the 
slot channel model is below $0.3 \mathrm{~mm}$ which is why the topology optimization improvement compared to the constrained case is higher than compared to the unconstrained case. The conductance improvement achieved by topology optimization is increasing with increasing effective thermal conductivity: a $1.7 \%$ conductance increase is observed for $0.5 \mathrm{~W} /(\mathrm{m} \mathrm{K})$ and a $71 \%$ increase with the fin width constraint and $36 \%$ increase without fin width constraint occurs for $300 \mathrm{~W} /(\mathrm{m} \mathrm{K})$. The increasing conductance improvement achieved with the topology optimized designs with increasing conductivity is due to the fact that designs with secondary branches from the main fins which differ more from the standard slot channel designs become more advantageous at higher conductivities than at low conductivities. Summarizing, it can be stated that the topology optimized designs afford a significant conductance improvement over the constraint slot channel model for roughly the same fin aspect ratio which can be used as a measure to estimate the printability of the designs. Also, relative to the unconstrained slot channel model the topology optimized designs show improved conductance. However, this is achieved with significantly lower aspect ratios of the fins; hence with topology optimized designs that are easier to print. Both observations in terms of conductance improvement and printability are most dramatic for higher effective polymer conductivities (above $10 \mathrm{~W} /(\mathrm{m} \mathrm{K})$ ).

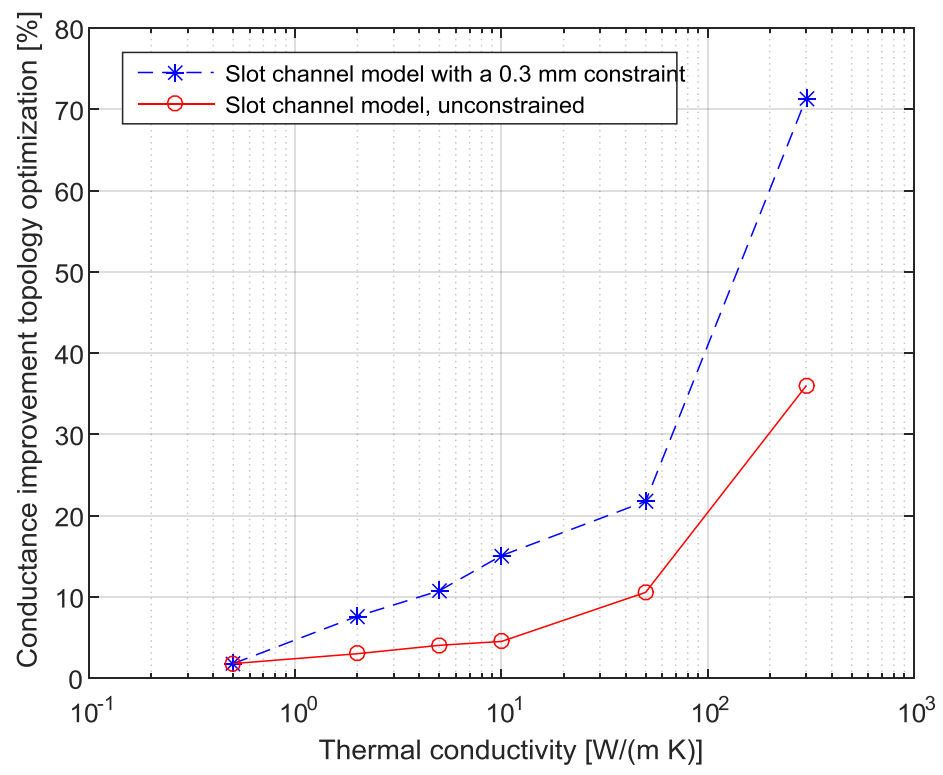

Fig. 12. Improvement of conductance per unit heat exchanger volume achieved with the topology optimized designs compared to the simple slot channel model plotted against the composite effective thermal conductivity. The cases of no minimum fin width in the slot channel model and a $0.3 \mathrm{~mm}$ minimum fin width in the slot channel model are considered.

\section{Conclusions}

This work addresses the air-side surface optimization of dry-cooled power plant condensers that are additively manufactured with polymers containing thermally conducting metal filaments. To tackle this design challenge, a fully developed flow thermofluid topology optimization model is developed and used to maximize the heat exchanger conductance. The fully developed flow assumption leads to simplified linear Navier-Stokes equations yielding a thermofluid topology optimization model that is stable for arbitrary Reynolds numbers within the 
laminar flow regime. This work demonstrates the applicability of thermofluid topology optimization to a real world heat exchanger design problem by using appropriate simplifications. Reynolds numbers of the flow in the optimized geometries are determined and the computed values are found to be significantly below the values of critical Reynolds numbers for laminar-turbulent transition reported in the literature. This confirms the laminar flow assumption that was used for the thermofluid modeling. Parametric studies are conducted to analyze the interplay of the heat exchanger material thermal conductivity and unit cell height on the system performance. The thermal conductivity is varied over three orders of magnitude and the influence of doubling and halving the pressure drop and temperature increase over the heat exchanger is studied using the same set of optimization parameters which demonstrates the robustness of the developed topology optimization model.

Apart from the obvious finding that higher filled polymer conductivities significantly increase the heat exchanger conductance, it is also shown that the optimal unit cell height increases with increasing conductivity of the filled polymer. Furthermore, it was found that the optimized designs tend to require finer feature sizes with increasing polymer conductivities. Hence, research should be conducted regarding the cost-efficient printability of small features in parallel with the development of 3D printable filled polymers with higher thermal conductivities. In future works, robust topology optimization approaches could be used to tailor the optimized designs to manufacturing constraints regarding the minimum printable solid feature size of the additive manufacturing process. To verify the developed topology optimization methodology, a comparison to a model using simple, slot-type air channels is conducted. It is shown that in terms of conductance per unit heat exchanger volume, the topology optimized designs outperform the simple slot geometry by up to $71 \%$ when the minimum fin width of the slot geometry is set to $0.3 \mathrm{~mm}$ and by up to $36 \%$ when an unconstrained slot geometry is considered. The conductance improvement through topology optimization increases with increasing effective polymer conductivity. To assess the printability of topology optimized designs and the slot channel designs, the fin aspect ratio, i.e. length over width of fins, is compared. The topology optimized designs show similar aspect ratios as the constraint slot-type geometry and, especially for higher conductivities, smaller aspect ratios, hence a better printability, than the unconstrained slot channel designs.

This work demonstrates that a significant performance improvement over simpler heat exchanger geometries can be obtained with the presented 2D optimization approach, thus confirming the usefulness of topology optimization to exploit the design freedom that additive manufacturing techniques provide. An experimental quantification of the impact of the fully developed flow assumption on the performance of the heat exchanger is planned in a future work; nevertheless, it should be noted that assuming a fully developed flow is a mature technique in thermofluid modeling. An interesting and industrially relevant extension of the presented modeling approach is the combination with existing thermofluid topology optimization models to allow for cross-flow heat exchanger design with explicit modeling of both fluids flowing perpendicular to each other.

\section{Acknowledgements}

This work was supported by the TOpTEn project sponsored through the Sapere Aude Program of the Danish Council for Independent Research (DFF - 4005-00320). Moreover, Jan Haertel would like to thank the Direktør, Professor H.I. Hannovers Legat for a travel grant that partially supported the research stay at UW-Madison during which the presented work was conducted. Professor Nellis gratefully acknowledges support from the 
Department of Energy Advanced Research Projects Agency through their ARID program (DE-FOA-0001197). Furthermore, the authors wish to thank Sanford Klein and Kurt Engelbrecht for the helpful discussions with regard to this work.

\section{Appendix A - Computation of Reynolds number in optimized designs}

It is customary to use the area mean velocity to characterize internal flow $[47,48]$ which is given by:

$w_{\text {mean }}=\frac{1}{A_{\mathrm{c}}} \int_{\mathrm{A}_{\mathrm{c}}} w d \mathrm{~A}_{c}$

where $\mathrm{A}_{\mathrm{c}}$ is the cross-sectional area of the flow. With $w_{\text {mean }}$ as characteristic velocity and using the thermophysical properties of air stated in Table 2, the Reynolds number in the optimized designs can be computed as follows:

$R e=\frac{\rho_{\text {air }} w_{\text {mean }} L_{\text {char }}}{\mu_{\text {air }}}$

where $L_{\text {char }}$ is a characteristic length of the geometry. However, it is not straightforward to define a characteristic length for the unconventional geometries of the topology optimized designs. Therefore, parts of the whole design are considered representatively for the entire design and compared to standard geometries for which the critical Reynolds number for laminar-turbulent transition is reported in the literature, i.e. pipe flow and flow between two parallel plates. This procedure is shown in the following exemplarily for the designs shown in Fig. A1: In both designs the largest flow area is identified that can be approximated by a rectangular duct (marked as orange rectangle) or by a flow between parallel plates (marked in white). It should be noted that the area used for the pipe flow approximation in Fig. 5 (f) is mirrored over the right boundary of the modeled domain which is not shown in the depiction. Similarly, the area used for the flow between two plates approximation is mirrored over the right boundary of the modeled domain in the design from Fig. 3 and mirrored over the bottom part of the modeled domain in the design from Fig. 5 (f). The characteristic length for the pipe flow approximation is the hydraulic diameter given by:

$D_{h}=\frac{2 a b}{a+b}$

where $a$ and $b$ are the respective length of the rectangle's sides. The characteristic length for the flow between two plates is the distance between the plates, i.e. two times the distance from the vertical white line to the right symmetry boundary in the design from Fig. 3 and two times the distance from the horizontal white line to the bottom boundary in the design from Fig. 5 (f). The mean velocity in the marked areas is computed in COMSOL according to Eq. (A.1). The results of these calculations are summarized in Table A.. Table A. contains additionally the mean velocity, characteristic length (i.e. width of air slots), and Reynolds number of the slot channel design from Fig. 10 (f). 

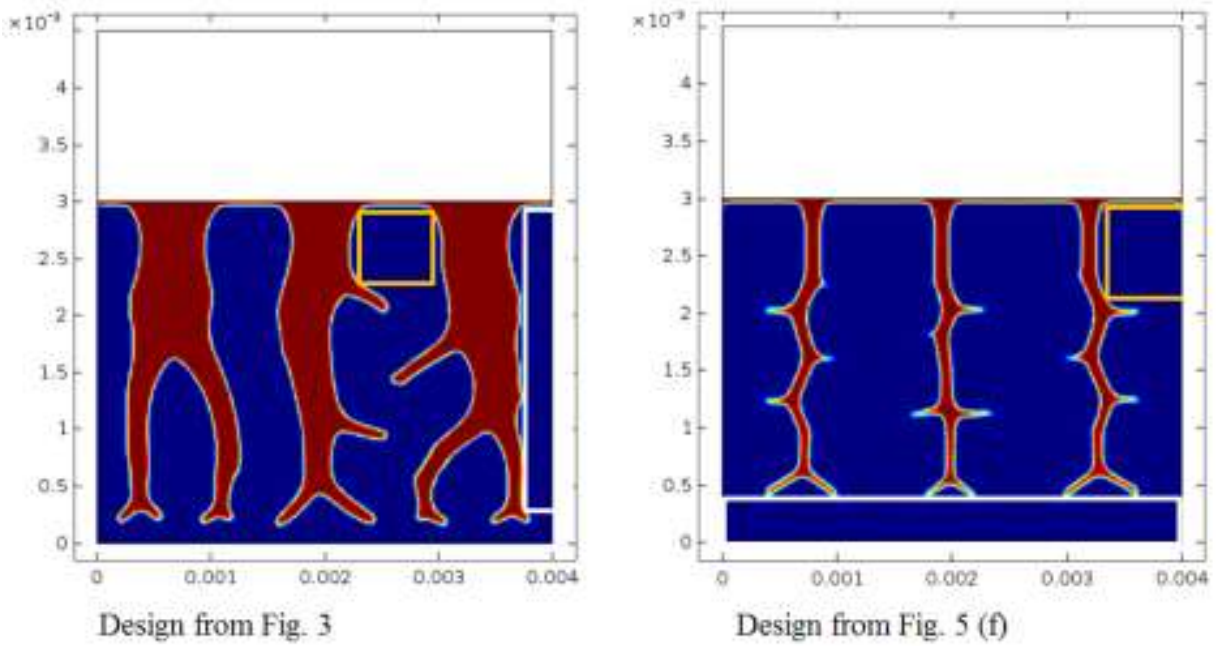

Fig. A1. Topology optimized designs from Fig. 3 and Fig. 5 (f). The areas in which the Reynolds number was determined assuming a pipe flow (orange) and flow between two parallel plates (white) are indicated.

Table A.1. Approximation of the Reynolds number in the topology optimized designs shown in Fig. A1 and the slot channel design from Fig. 9 (f). The flow conditions in the topology optimized designs are approximated assuming a pipe flow in a rectangular duct as well as a flow between two parallel plates.

\begin{tabular}{lllll}
\hline Design & Considered flow & $w_{\text {mean }}[\mathrm{m} / \mathrm{s}]$ & $L_{\text {char }}[\mathrm{mm}]$ & $R e[-]$ \\
\hline Topology optimized design & Pipe flow & 7.4 & 0.64 & 271 \\
from Fig. 3 & Parallel plates & 7.6 & 0.73 & 318 \\
Topology optimized design & Pipe flow & 10.7 & 1.00 & 614 \\
from Fig. 5 (f) & Parallel plates & 14.0 & 0.80 & 643 \\
Slot channel design from Fig. & Parallel plates & 7.0 & 0.64 & 257 \\
10 (f) & & & \\
\hline
\end{tabular}

\section{Appendix B - Nusselt number comparison to analytical solution}

For two simple geometries, i.e. flow between two parallel plates and flow in a circular pipe, the Nusselt number obtained from the numerical validation model is compared to the respective analytical solution. As stated in section 4.1, only the heat transfer problem in the air flow is modeled for these geometries and the temperature at the boundary to the solid is set to the fixed value $T_{\text {wall }}$. The same values as in the optimization model are used for the pressure drop and temperature increase over the heat exchanger (stated in Table 2) and $T_{\text {wall }}$ is set to the same value as $T_{\text {water }}$ in the optimization model since the heat conduction in the solid is not considered in the validation model. The characteristic dimension of the validation model, i.e. the distance between the parallel plates or the diameter of the circular pipe, is adjusted so that $T_{\text {air,bulk takes the value of }}$ $T_{\text {air }, \text { bulk }}^{*}$ stated in Table 2. Thus, the problem modeled in the validation model is similar to the original problem of the optimization model. 
The mean Nusselt number in the context of the considered internal flow problems is defined as:

$N u=\frac{h D_{h}}{k_{\text {air }}}$

where $h$ is the average heat transfer coefficient within the duct. The hydraulic diameter $D_{h}$ corresponds to the pipe diameter in the case of flow inside a circular pipe and two times the distance of the plates in the case of flow between parallel plates. The heat transfer coefficient in the 2D fully developed flow model is given by:

$h=\frac{\dot{q}}{\operatorname{per}\left(T_{\text {wall }}-T_{\text {air }, \text { bulk }}\right)}$

where per is the perimeter of the duct which can be computed by:

per $=2$ height $L \quad$ for flow between parallel plates

per $=\pi D L \quad$ for flow in a circular pipe

where $D$ is the pipe diameter and height is the modeled height of the flow between parallel plates problem. The height modeled is set to $4 \mathrm{~mm}$ and symmetry conditions are applied at the top and bottom of the modeled domain to represent an infinitely extended duct perpendicular to the air flow direction. Using the above equations, the Nusselt number in the numerical fully developed flow model can be computed. This Nusselt number is equivalent to the Nusselt number in a fully developed flow problem with constant wall heat flux in the axial direction, for which analytical solutions exist, as described in section 4.1. The comparison between the Nusselt number computed using the numerical model according to equation (B.1) and the respective analytical solution for the case of flow between parallel plates [50] and flow in a circular pipe [51] is shown in Table B.1.

Table B.1. Thermally and fluid dynamically fully developed flow Nusselt number and hydraulic diameter for flow between parallel plates and flow in a circular pipe obtained from the numerical model and from analytical solutions in the literature.

\begin{tabular}{llll}
\hline Geometry & Case & $D_{h}[\mathrm{~mm}]$ & $N u[-]$ \\
\hline Flow between parallel plates & Analytical solution [50] & - & 8.235 \\
& Numerical model & 0.67 & 8.241 \\
Flow in a circular pipe & Analytical solution [51] & - & 4.364 \\
& Numerical model & 0.52 & 4.367 \\
\hline
\end{tabular}

\section{Nomenclature}

$a$

$A_{\Omega, f l}$

$A_{c}$

$b$
Side length of rectangle for Reynolds number calculation [m]

Flow area of design domain $\left[\mathrm{m}^{2}\right]$

Cross-sectional area $\left[\mathrm{m}^{2}\right]$

Side length of rectangle for Reynolds number calculation [m] 


\begin{tabular}{|c|c|}
\hline$b_{k}$ & Convexity parameter in RAMP interpolation \\
\hline$c$ & Specific heat capacity $[\mathrm{J} /(\mathrm{kg} \mathrm{K})]$ \\
\hline$D$ & Diameter of circular pipe $[\mathrm{m}]$ \\
\hline$D_{h}$ & Hydraulic diameter $[\mathrm{m}]$ \\
\hline$F$ & Brinkman friction term $\left[\mathrm{N} / \mathrm{m}^{3}\right]$ \\
\hline$\dot{g}_{v}^{\prime \prime \prime}$ & Volumetric rate of viscous dissipation $\left[\mathrm{W} / \mathrm{m}^{3}\right]$ \\
\hline$h$ & Heat transfer coefficient $\left[\mathrm{W} /\left(\mathrm{m}^{2} \mathrm{~K}\right)\right]$ \\
\hline height & Modeled height of flow between parallel plates problem [m] \\
\hline$k$ & Thermal conductivity $[\mathrm{W} /(\mathrm{m} \mathrm{K})]$ \\
\hline$L$ & Length heat exchanger unit cell $[\mathrm{m}]$ \\
\hline$L_{\text {char }}$ & Characteristic length $[\mathrm{m}]$ \\
\hline $\mathbf{n}$ & Normal vector \\
\hline$n_{d}$ & Number of design variables \\
\hline$N u$ & Nusselt number [-] \\
\hline$p$ & Pressure $[\mathrm{Pa}]$ \\
\hline per & Perimeter of duct $[\mathrm{m}]$ \\
\hline$\dot{q}$ & Heat transfer rate $[\mathrm{W}]$ \\
\hline$r$ & Residual of the state problem \\
\hline$r_{\text {filter }}$ & Filter parameter $[\mathrm{m}]$ \\
\hline $\operatorname{Re}$ & Reynolds number \\
\hline$s$ & Vector of state variables of the multiphysics problem \\
\hline$T$ & Temperature $\left[{ }^{\circ} \mathrm{C}\right]$ \\
\hline$T_{\text {air,bulk }}$ & Air bulk temperature $\left[{ }^{\circ} \mathrm{C}\right]$ \\
\hline$T_{\text {air }, \text { bulk }}^{*}$ & Prescribed air bulk temperature $\left[{ }^{\circ} \mathrm{C}\right]$ \\
\hline$T_{\text {wall }}$ & Wall temperature of duct $\left[{ }^{\circ} \mathrm{C}\right]$ \\
\hline$U A$ & Heat exchanger's conductance $[\mathrm{kW} / \mathrm{K}]$ \\
\hline$w$ & Velocity in z-direction $[\mathrm{m} / \mathrm{s}]$ \\
\hline$w_{\text {average, } f l}$ & Flow area averaged air velocity $[\mathrm{m} / \mathrm{s}]$ \\
\hline$w_{\text {mean }}$ & Mean velocity used for Reynolds number calculation $[\mathrm{m} / \mathrm{s}]$ \\
\hline $\mathrm{W}$ & Wall domain as specified in Fig. 2 \\
\hline $\mathrm{x}$ & $\mathrm{x}$-coordinate $[\mathrm{m}]$ \\
\hline $\mathrm{y}$ & $\mathrm{y}$-coordinate $[\mathrm{m}]$ \\
\hline $\mathrm{z}$ & $\mathrm{z}$-coordinate $[\mathrm{m}]$ \\
\hline
\end{tabular}


Abbreviations

2D

3D

ABS

$\mathrm{BC}$

FLM

GCMMA

$\max$

PDE

s.t.

Greek symbols

$\bar{\alpha}$

$\beta$

$\gamma$

$\gamma$

$\Gamma_{\mathrm{ij}}$

$\partial$

$\triangle p_{H E X}$

$\triangle T$

$\triangle T_{H E X}$

$\eta$

$\mu$

$\nabla$

$\rho$

$\Omega$

Indices

air

f

i

$\mathrm{j}$
Two-dimensional

Three-dimensional

Acrylonitrile butadiene styrene

Boundary condition

Fused layer modeling

Globally convergent version of the Method of Moving Asymptotes

Maximize

Partial differential equation

Subject to

Maximum inverse permeability $\left[\mathrm{Pa} \mathrm{s} / \mathrm{m}^{2}\right]$

Projection steepness parameter

Design variable

Vector of design variables

Boundary of modeled domain as specified in Fig. 2

Partial derivative

Air-side pressure drop over the heat exchanger unit cell [Pa]

Air-to-water temperature difference $\left[{ }^{\circ} \mathrm{C}\right]$

Air-side temperature increase across the heat exchanger unit cell $\left[{ }^{\circ} \mathrm{C}\right]$

Projection threshold parameter

Dynamic viscosity [Pa s]

Nabla operator

Density $\left[\mathrm{kg} / \mathrm{m}^{3}\right]$

Design domain as specified in Fig. 2

Air

Fluid

Counter index

Counter index 


$\begin{array}{ll}\text { polymer } & \text { Polymer } \\ \mathrm{s} & \text { Solid } \\ \text { water } & \text { Water }\end{array}$

\section{Literature}

[1] Macknick, J., Newmark, R., Heath, G., \& Hallett, K. C. (2011). A review of operational water consumption and withdrawal factors for electricity generating technologies. technical report NREL/TP6A20-50900, National Renewable Energy Laboratory, http://dx.doi.org/10.2172/1219149

[2] T'Joen, C., Park, Y., Wang, Q., Sommers, A., Han, X., \& Jacobi, A. (2009). A review on polymer heat exchangers for HVAC\&R applications. International Journal of Refrigeration, 32(5), 763-779, http://dx.doi.org/10.1016/j.ijrefrig.2008.11.008

[3] Bendsøe, M. P., \& Sigmund, O. (2013). Topology optimization: theory, methods, and applications. Springer Science \& Business Media, http://dx.doi.org/10.1007/978-3-662-05086-6

[4] Borrvall, T., \& Petersson, J. (2003). Topology optimization of fluids in Stokes flow. International journal for numerical methods in fluids, 41(1), 77-107, http://dx.doi.org/10.1002/fld.426

[5] Jensen, J. S., \& Sigmund, O. (2011). Topology optimization for nano-photonics. Laser \& Photonics Reviews, 5(2), 308-321, http://dx.doi.org/10.1002/lpor.201000014

[6] Li, Q., Steven, G. P., Xie, Y. M., \& Querin, O. M. (2004). Evolutionary topology optimization for temperature reduction of heat conducting fields. International Journal of Heat and Mass Transfer, 47(23), 5071-5083, http://dx.doi.org/10.1016/j.ijheatmasstransfer.2004.06.010

[7] Gersborg-Hansen, A., Bendsøe, M. P., \& Sigmund, O. (2006). Topology optimization of heat conduction problems using the finite volume method. Structural and multidisciplinary optimization, 31(4), 251-259, http://dx.doi.org/10.1007/s00158-005-0584-3

[8] Sigmund, O. (2001). Design of multiphysics actuators using topology optimization-Part I: Onematerial structures. Computer methods in applied mechanics and engineering, 190(49), 6577-6604, http://dx.doi.org/10.1016/s0045-7825(01)00251-1

[9] Yin, L., \& Ananthasuresh, G. K. (2002). A novel topology design scheme for the multi-physics problems of electro-thermally actuated compliant micromechanisms. Sensors and Actuators A: Physical, 97, 599-609, http://dx.doi.org/10.1007/978-3-642-59497-7 59

[10] Bruns, T. E. (2007). Topology optimization of convection-dominated, steady-state heat transfer problems. International Journal of Heat and Mass Transfer, 50(15), 2859-2873, http://dx.doi.org/10.1016/j.ijheatmasstransfer.2007.01.039

[11] Ahn, S. H., \& Cho, S. (2010). Level set-based topological shape optimization of heat conduction problems considering design-dependent convection boundary. Numerical Heat Transfer, Part B: Fundamentals, 58(5), 304-322, http://dx.doi.org/10.1080/10407790.2010.522869

[12] Iga, A., Nishiwaki, S., Izui, K., \& Yoshimura, M. (2009). Topology optimization for thermal conductors considering design-dependent effects, including heat conduction and convection. International Journal of Heat and Mass Transfer, 52(11), 2721-2732, http://dx.doi.org/10.1016/j.ijheatmasstransfer.2008.12.013 
[13] Zhou, M., Alexandersen, J., Sigmund, O., \& Pedersen, C. B. (2016). Industrial application of topology optimization for combined conductive and convective heat transfer problems. Structural and Multidisciplinary Optimization, 1-16, http://dx.doi.org/10.1007/s00158-016-1433-2

[14] Coffin, P., \& Maute, K. (2016). Level set topology optimization of cooling and heating devices using a simplified convection model. Structural and Multidisciplinary Optimization, 53(5), 985-1003, http://dx.doi.org/10.1007/s00158-015-1343-8

[15] Soprani, S., Haertel, J. H. K., Engelbrecht, K., Lazarov, B. S., \& Sigmund, O. (2015). Topology Optimization of an Actively Cooled Electronics Section for Downhole Tools. In Proceedings. Comsol Conference 2015.

[16] Soprani, S., Haertel, J. H. K., Lazarov, B. S., Sigmund, O., \& Engelbrecht, K. (2016). A design approach for integrating thermoelectric devices using topology optimization. Applied Energy, 176, 4964, http://dx.doi.org/10.1016/j.apenergy.2016.05.024

[17] Dede, E. M. (2009). Multiphysics topology optimization of heat transfer and fluid flow systems. In proceedings of the COMSOL Users Conference.

[18] Yoon, G. H. (2010). Topological design of heat dissipating structure with forced convective heat transfer. Journal of Mechanical Science and Technology, 24(6), 1225-1233, http://dx.doi.org/10.1007/s12206-010-0328-1

[19] Dede, E. M. (2012). Optimization and design of a multipass branching microchannel heat sink for electronics cooling. Journal of Electronic Packaging, 134(4), 041001, http://dx.doi.org/10.1115/1.4007159

[20] Van Oevelen, T., \& Baelmans, M. (2014). Numerical topology optimization of heat sinks. In Proceedings of the 15th International Heat Transfer Conference, 10-15, Begell House Publishers, http://dx.doi.org/10.1615/ihtc15.opt.009168

[21] Marck, G., Nemer, M., \& Harion, J. L. (2013). Topology optimization of heat and mass transfer problems: laminar flow. Numerical Heat Transfer, Part B: Fundamentals, 63(6), 508-539, http://dx.doi.org/10.1080/10407790.2013.772001

[22] Matsumori, T., Kondoh, T., Kawamoto, A., \& Nomura, T. (2013). Topology optimization for fluidthermal interaction problems under constant input power. Structural and Multidisciplinary Optimization, 47(4), 571-581, http://dx.doi.org/10.1007/s00158-013-0887-8

[23] Yaji, K., Yamada, T., Yoshino, M., Matsumoto, T., Izui, K., \& Nishiwaki, S. (2016). Topology optimization in thermal-fluid flow using the lattice Boltzmann method. Journal of Computational Physics, 307, 355-377, http://dx.doi.org/10.1016/j.jcp.2015.12.008

[24] McConnell, C., \& Pingen, G. (2012). Multi-Layer, Pseudo 3D Thermal Topology Optimization of Heat Sinks. ASME 2012 International Mechanical Engineering Congress and Exposition, Houston, Texas, USA, http://dx.doi.org/10.1115/imece2012-93093

[25] Haertel, J. H. K., Engelbrecht, K., Lazarov, B. S., \& Sigmund, O. (2015). Topology Optimization of Thermal Heat Sinks. In Proceedings. Comsol Conference 2015.

[26] Qian, X., \& Dede, E. M. (2015). Topology optimization of a coupled thermal-fluid system under a tangential thermal gradient constraint. Structural and Multidisciplinary Optimization, 1-21, http://dx.doi.org/10.1007/s00158-016-1421-6

[27] Kontoleontos, E. A., Papoutsis-Kiachagias, E. M., Zymaris, A. S., Papadimitriou, D. I., \& Giannakoglou, K. C. (2013). Adjoint-based constrained topology optimization for viscous flows, 
including heat transfer. Engineering Optimization, 45(8), 941-961, https://doi.org/10.1080/0305215x.2012.717074

[28] Koga, A. A., Lopes, E. C. C., Nova, H. F. V., de Lima, C. R., \& Silva, E. C. N. (2013). Development of heat sink device by using topology optimization. International Journal of Heat and Mass Transfer, 64, 759-772, http://dx.doi.org/10.1016/j.ijheatmasstransfer.2013.05.007

[29] Yaji, K., Yamada, T., Kubo, S., Izui, K., \& Nishiwaki, S. (2015). A topology optimization method for a coupled thermal-fluid problem using level set boundary expressions. International Journal of Heat and Mass Transfer, 81, 878-888, http://dx.doi.org/10.1016/j.ijheatmasstransfer.2014.11.005

[30] Alexandersen, J., Aage, N., Andreasen, C. S., \& Sigmund, O. (2014). Topology optimisation for natural convection problems. International Journal for Numerical Methods in Fluids, 76(10), 699-721, http://dx.doi.org/10.1002/fld.3954

[31] Alexandersen, J. (2015). Topology optimisation of passive coolers for light-emitting diode lamps. In 11th World Congress of Structural and Multidisciplinary Optimisation.

[32] Alexandersen, J., Sigmund, O., \& Aage, N. (2016). Large scale three-dimensional topology optimisation of heat sinks cooled by natural convection. International Journal of Heat and Mass Transfer, 100, 876-891, http://dx.doi.org/10.1016/j.ijheatmasstransfer.2016.05.013

[33] Coffin, P., \& Maute, K. (2016). A level-set method for steady-state and transient natural convection problems. Structural and Multidisciplinary Optimization, 53(5), 1047-1067, http://dx.doi.org/10.1007/s00158-015-1377-y

[34] Dede, E. M., Joshi, S. N., \& Zhou, F. (2015). Topology optimization, additive layer manufacturing, and experimental testing of an air-cooled heat sink. Journal of Mechanical Design, 137(11), 111403, http://dx.doi.org/10.1115/1.4030989

[35] Castro, D. A., Kiyono, C. Y., \& Silva, E. C. N. (2015). Design of radiative enclosures by using topology optimization. International Journal of Heat and Mass Transfer, 88, 880-890, http://dx.doi.org/10.1016/j.ijheatmasstransfer.2015.04.077

[36] Dbouk, T. (2017). A review about the engineering design of optimal heat transfer systems using topology optimization. Applied Thermal Engineering, 112, 841-854, http://dx.doi.org/10.1016/j.applthermaleng.2016.10.134

[37] Doodman, A. R., Fesanghary, M., \& Hosseini, R. (2009). A robust stochastic approach for design optimization of air cooled heat exchangers. Applied Energy, 86(7), 1240-1245. https://doi.org/10.1016/j.apenergy.2008.08.021

[38] Bhutta, M. M. A., Hayat, N., Bashir, M. H., Khan, A. R., Ahmad, K. N., \& Khan, S. (2012). CFD applications in various heat exchangers design: A review. Applied Thermal Engineering, 32, 1-12. https://doi.org/10.1016/j.applthermaleng.2011.09.001

[39] Nellis, G., \& Klein, S. (2008). Heat Transfer, Cambridge University Press. New York, http://dx.doi.org/10.1017/cbo9780511841606

[40] Stolpe, M., \& Svanberg, K. (2001). An alternative interpolation scheme for minimum compliance topology optimization. Structural and Multidisciplinary Optimization, 22(2), 116-124, http://dx.doi.org/10.1007/s001580100129

[41] Sigmund, O., \& Petersson, J. (1998). Numerical instabilities in topology optimization: a survey on procedures dealing with checkerboards, mesh-dependencies and local minima. Structural optimization, 16(1), 68-75, http://dx.doi.org/10.1007/bf01214002 
[42] Lazarov, B. S., \& Sigmund, O. (2011). Filters in topology optimization based on Helmholtz-type differential equations. International Journal for Numerical Methods in Engineering, 86(6), 765-781, http://dx.doi.org/10.1002/nme.3072

[43] Wang, F., Lazarov, B. S., \& Sigmund, O. (2011). On projection methods, convergence and robust formulations in topology optimization. Structural and Multidisciplinary Optimization, 43(6), 767-784, http://dx.doi.org/10.1007/s00158-010-0602-y

[44] COMSOL Multiphysics 5.2

[45] Svanberg, K. (2002). A class of globally convergent optimization methods based on conservative convex separable approximations. SIAM journal on optimization, 12(2), 555-573, http://dx.doi.org/10.1137/s1052623499362822

[46] Felber, R., Nellis, G., Rudolph, N. (2016). Design and Modeling of 3D Printed Air-Cooled Heat Exchangers. In Proceedings of the 16th International Refrigeration and Air Conditioning Conference at Purdue

[47] Avila, K., Moxey, D., de Lozar, A., Avila, M., Barkley, D., \& Hof, B. (2011). The onset of turbulence in pipe flow. Science, 333(6039), 192-196, https://doi.org/10.1126/science.1203223

[48] Hanks, R. W. (1963). The laminar-turbulent transition for flow in pipes, concentric annuli, and parallel plates. AIChE Journal, 9(1), 45-48, https://doi.org/10.1002/aic.690090110

[49] Lazarov, B. S., Wang, F., \& Sigmund, O. (2016). Length scale and manufacturability in density-based topology optimization. Archive of Applied Mechanics, 86(1-2), 189-218. http://dx.doi:10.1007/s00419$\underline{015-1106-4}$

[50] Cess, R. D., \& Shaffer, E. C. (1959). Heat transfer to laminar flow between parallel plates with a prescribed wall heat flux. Applied Scientific Research, Section A, 8(1), 339-344. https://doi.org/10.1007/bf00411758

[51] Siegel, R., Sparrow, E. M., \& Hallman, T. M. (1958). Steady laminar heat transfer in a circular tube with prescribed wall heat flux. Applied Scientific Research, Section A, 7(5), 386-392.

[52] Bergman, T. L., Incropera, F. P., DeWitt, D. P., \& Lavine, A. S. (2011). Fundamentals of heat and mass transfer. John Wiley \& Sons.

[53] Hruska, P. J. (2016). Exploring Dry Air Cooling Heat Exchanger Technologies and Applications, Master thesis, University of Wisconsin - Madison. 